\title{
Evaporation of sessile drops: A 3D approach
}

\author{
P. J. Sáenz ${ }^{1}$, K. Sefiane ${ }^{1,2}$, J. Kim ${ }^{3}$, O. K. Matar ${ }^{4}$ and P. Valluri ${ }^{1} \dagger$ \\ ${ }^{1}$ Institute for Materials and Processes, The University of Edinburgh, Edinburgh, EH9 3JL, UK \\ ${ }^{2}$ International Institute for Carbon-Neutral Energy Research (I2CNER), Kyushu University, \\ Fukuoka, 819-0395, Japan \\ ${ }^{3}$ Dept. of Mechanical Engineering, The University of Maryland, College Park, MD 20742, USA \\ ${ }^{4}$ Dept. of Chemical Engineering, Imperial College London, London, SW7 2AZ, UK
}

(Received ?; revised ?; accepted ?. - To be entered by editorial office)

The evaporation of non-axisymmetric sessile drops is studied by means of experiments and three-dimensional (3D) direct numerical simulations. The emergence of azimuthal currents and pairs of counter-rotating vortices in the liquid bulk flow is reported in drops with non-circular contact area. These phenomena, especially the latter which is also observed experimentally, are found to play a critical role in the transient flow dynamics and associated heat transfer. Non-circular drops exhibit variable wettability along the pinned contact-line sensitive to the choice of system parameters, and inversely dependent on the local contact-line curvature, providing a simple criterion for estimating the approximate contact-angle distribution. The evaporation rate is found to vary in the same order of magnitude as the liquid-gas interfacial area. Furthermore, the more complex case of drops evaporating with a moving contact line in the constant contact-angle mode is addressed. Interestingly, the numerical results demonstrate that the average interface temperature remains essentially constant as the drop evaporates in the constant-angle mode, while this increases in the constant-radius mode as the drops becomes thinner. It is therefore concluded that, for increasing substrate heating, the evaporation rate increases more rapidly in the constant-radius mode than in the constant-angle mode. In other words, the higher the temperature the larger the difference between the lifetimes of an evaporating drop in the constant-angle mode with respect to that evaporating in the constant-radius mode.

Key words: evaporating drop, 3D, moving contact line, diffuse-interface method

\section{Introduction}

The evaporation of a liquid droplet on a solid substrate is a common physical phenomenon encountered in a vast variety of situations; it is, therefore, unsurprising that it has received significant attention in the literature. For this reason, only the publications most relevant to the subject of the present work will be highlighted here; the reader is referred to Dunn et al. (2009); Cazabat \& Guena (2010); Erbil (2012) for other recent and comprehensive reviews of the topic. In addition, the discussion will be restricted to investigations of relatively slow evaporating drops in which the surface remains (quasi) saturated; the transport of vapour away from the drop is the rate-limiting mass-transfer mechanism. It should be noted, nonetheless, that there is another body of work concerning the complementary situation of more rapidly evaporating liquids showing ther-

$\dagger$ Email address for correspondence: prashant.valluri@ed.ac.uk 
modynamic discontinuities at the interface (Fang \& Ward 1999; Ward \& Fang 1999). Representative investigations are those by Burelbach et al. (1988); Anderson \& Davis (1995); Ajaev (2005).

A milestone investigation on sessile drops was performed by Picknett \& Bexon (1977) who experimentally and theoretically examined this problem in the late 70s. They addressed the two extreme modes of evaporation, namely "constant angle" (CA) and "constant radius" (CR) modes. In the former, the droplet evaporates with a receding contact radius $R$ while the contact angle remains fixed $\theta=\theta_{0}$. In the latter, the contact angle $\theta$ decreases with time while the base radius remains pinned $R=R_{0}$. Picknett \& Bexon (1977) observed that the drop mass varies linearly, and according to a power-law when the drop evaporates in the CR and CA modes, respectively. They also examined the lifetime of the drop as a function of the initial (equilibrium) contact angle, $\theta_{0}$, concluding that, in general, for the same $\theta_{0}$, the lifetime of a drop evaporating in the CR mode is shorter than that of same drop evaporating in the CA mode. Only when the level of hydrophobicity is very high, $\theta>140^{\circ}$, is this behaviour inverted. Bourgès-Monnier \& Shanahan (1995) conducted experiments to study the impact of the substrate roughness and showed that a droplet could also evaporate in a more complicated fashion mixing $\mathrm{CR}$ and CA modes. This mechanism is usually referred to as stick-slip (SS) mode. They focused their attention on the CR stage, and obtained an approximate analytical solution for the total mass flux across the droplet surface.

Significant insights into the drop dynamics were provided by Deegan et al. (1997, 2000) in their investigation of the so-called "coffee-ring" effect, i.e the patterns left by pinned drops when the evaporating liquid contains a suspension of colloidal particles. This was explained via the radial outward flow induced to replenish the liquid eliminated by evaporation from the edge of the drop. Hu \& Larson (2002) adopted the evaporation flux distribution along the droplet surface presented by Deegan et al. (2000). With the help of their numerical results, they suggested approximate analytical expressions to determine the temporal evolution of the evaporation rate as a function of the contact angle $\theta$, in the range $0<\theta<90^{\circ}$. The authors compared their results with experiments, numerical simulations, and the theory by Picknett \& Bexon (1977) finding reasonably good agreement without parameter fitting. Subsequently, $\mathrm{Hu} \&$ Larson $(2005 a, b)$ neglected inertial effects and employed lubrication theory to investigate the flow field within the drops, in the absence ( $\mathrm{Hu} \&$ Larson 2005b) and presence (Hu \& Larson 2005a) of Marangoni stresses. The lubrication approximation restricted the validity of their analysis to $\theta<40^{\circ}$. Following on from these works, Hu \& Larson (2006) also showed that spontaneous, evaporation-induced thermocapillarity could invert the typical coffee-ring depositions, therefore, driving the suspended particles to the center of the drop rather than towards the contact line. Ristenpart et al. (2007) concluded that the direction of the flow depends on the relative thermal conductivities of the substrate and liquid, and provided the critical conditions that demarcate the outward/inward flow regimes.

Other works important to the subject of this investigation are those by Birdi et al. (1989) and Rowan et al. (1995) who conducted experiments showing that the evaporation rate varies with the size of the drops. Erbil et al. (2002) observed the CA evaporation mode, which is normally more unusual, in n-butanol, toluene, n-nonane, and n-octane drops on a polytetrafluoroethylene (PTFE) surface with equilibrium contact angle $\theta_{0}<$ $90^{\circ}$. More work on evaporating drops of pure, completely wetting fluids with receding contact lines was later performed by Poulard et al. (2003). Under such conditions, they observed that the dynamics are controlled not only by the volatility of the liquid, but also by the properties of the wetting film left on the substrate. The role of the substrate temperature on the wetting and evaporation behaviour of volatile droplets was examined 
by Crafton \& Black (2004), with water and heptane drops on aluminium and copper surfaces, and by Mollaret et al. (2004), with water drops on aluminium and PTFE. David et al. (2007) demonstrated a strong influence of the substrate's thermal conductivity on the evaporation rate. This important finding pointed out deficiencies of previous theoretical models (Picknett \& Bexon 1977; Bourgès-Monnier \& Shanahan 1995; Rowan et al. 1995; $\mathrm{Hu} \&$ Larson 2002), which failed to take this into account. Later, Dunn et al. (2009) provided further insights into this problem by means of experiments and numerical work.

New theoretical expressions to predict the evaporation rate correcting the deviations due to thermal effects have been recently presented by Sefiane \& Bennacer (2011), and Sobac \& Brutin (2012). Xu \& Luo (2007) showed a weak Marangoni flow experienced by water drops via fluorescent nanoparticles added to the liquid. Sefiane et al. (2009) performed experiments to elucidate the effect of the atmosphere on pinned water drops released onto various substrates. They observed that reducing the atmospheric pressure increased the molecular diffusion coefficient of the vapour and, therefore, the evaporation rate. Different evaporation rates were also reported for different ambient gases, namely nitrogen, helium, and carbon dioxide. Shanahan et al. (2011) revised the CA and CR pure modes of evaporation and derived new analytical expressions to demonstrate that the droplet lifetime changes with the hydrophobicity of the substrate. As Picknett \& Bexon (1977), they also concluded that the CR mode leads to shorter drop lifetimes. In an attempt to investigate the more complex SS mode, Nguyen \& Nguyen (2012) proposed a more sophisticated theoretical analysis to predict the lifetime of droplets evaporating via a combined pinned-receding mode. Under these conditions, Stauber et al. (2014) have recently proposed a master diagram for the lifetimes of drops in all possible modes showing that the lifetime of a drop may not always be constrained by the lifetimes of the extreme modes.

A number of experimental investigations have also revolved around the complex pinningdepinning behaviour of the triple line (SS mode), e.g. Sefiane \& Tadrist (2006), Moffat et al. (2009), and Orejon et al. (2011). The dynamics of this are dictated by a competition between pinning forces on one hand and depinning forces on the other. The former are usually due to the contact line being anchored to the substrate because of chemical and surface heterogeneities; the depinning forces are normally the result of the deviation of the droplet profile from equilibrium (Orejon et al. 2011). Before these works, Shanahan (1995) had already developed a simple theory to explain the jumps characteristic of the SS mode experienced by the drops in the last stages of the evaporation process. Recently, Jansen et al. (2014) examined elongated droplets on chemically stripepatterned surfaces. The contact-line dynamics of these drops are intimately connected with the pattern's characteristic direction. After an initial stage wherein the whole contract line is motionless, these drops evaporate with fragments of its contact line pinned (segments parallel to the stripe pattern) while the rest moves (segments perpendicular to the pattern).

Most of the aforementioned theoretical works essentially focus on predicting the evaporation flux and lifetime of the drops regardless of their internal dynamics, except for Deegan et al. (1997, 2000); Hu \& Larson (2005a,b, 2006); Mollaret et al. (2004); Karapetsas et al. (2012) who also provide some insights into the dynamics of bulk flow via numerical simulations. Other authors have also directed their attention to revealing the underlying mechanisms taking place within the liquid, e.g. Ruiz \& Black (2002); Girard et al. (2006, 2008a,b); Girard \& Antoni (2008). At this point, it is important to realize the restrictions these numerical works share. Firstly, most of these investigation completely neglected the dynamics of the gas phase by addressing the free-surface problem. 
In some cases, the authors use empirical heat-transfer coefficients to approximate the energy balance. Only Girard et al. (2008a,b); Girard \& Antoni (2008) solve Laplace's equation for the temperature in the gas phase to calculate more realistically the energy transfer across the free surface. Regarding the evaporation flux, this is computed either via the Laplace solution of the vapour field (Mollaret et al. 2004; Hu \& Larson 2002, 2005a,b, 2006), or via use of an empirical mass-transfer coefficient (Ruiz \& Black 2002; Girard et al. 2006, 2008a,b; Girard \& Antoni 2008; Karapetsas et al. 2012). In thermocapillary flows, however, the Marangoni effect may induce significant convective transport of vapour in the gas parallel to the interface which can alter the evaporation flux distribution as was shown by Sáenz et al. (2014). Thus, the full advection-diffusion solution for the vapour concentration (and temperature) in the gas is necessary to verify how the resulting local interface mass-transfer rate (calculated purely based on a local temperature/concentration balance at the interface) compares with that from the solution of the diffusion equation.

The reader should also note that relevant works available in the literature, such as $\mathrm{Hu}$ \& Larson (2002, 2005a,b, 2006) or Girard et al. (2006, 2008a,b); Girard \& Antoni (2008), provide pseudo-transient solutions to the droplet development in time, i.e. their transient solution is the sum of a set of steady-state solutions rather than the result of a pure transient approach as those by Ruiz \& Black (2002); Mollaret et al. (2004); Karapetsas et al. (2012). Pseudo-transient works additionally require the external imposition of the drop geometry. Thus, these models are not well-suited to capturing transient phenomena, e.g. themocapillary instabilities as those recently discovered by Sefiane et al. (2008), nor interfacial deformations, e.g. for larger drops in which gravity is not negligible as those investigated by Gatapova et al. (2014). Very recently, Yang et al. (2014) conducted finiteelement simulations of a fully-coupled three-phase model (solid-liquid-gas), restricted to 2D, to describe the liquid and gas flow with and without Marangoni effects. They analyzed the non-heated problem and reported an increasing evaporation rate due to the Marangoni effect.

The simulation of evaporating drops with a moving contact line (MCL) has been undertaken by Murisic \& Kondic (2011) and Karapetsas et al. (2012) via the "one-sided" approach. Murisic \& Kondic (2011) investigated the two traditional evaporation models (equilibrium and non-equilibrium interface) with the help of the lubrication approximation, which limited their work to $\theta<40^{\circ}$. Depending on the model, they found significantly different results, including drop evolution and thermal gradients along the liquid-gas interface. Karapetsas et al. (2012) conducted simulations providing insights for a mixed pinned-receding scenario. Focusing on rather thin drops, $\theta<23^{\circ}$, they reported spontaneous emergence of unstable convective rolls in the 2D axisymetric flow for increasing Prandtl number. As pointed out by Sui et al. (2014) in their recent review of the state of the art in the numerical simulation of flows with a MCL, the direct numerical simulation (DNS) of a fully-coupled two-phase flow with an MCL and phase-change remains a challenge. In addition, it is important to develop a model capable of resolving both pure $\mathrm{CR}$ and $\mathrm{CA}$ evaporation modes to examine how the dynamics compare for the extreme cases, similarly to what Picknett \& Bexon (1977) did with the overall evaporation rate.

All of the aforementioned numerical and theoretical work has been concerned with axisymmetric drops, with the exception of the linear-stability analysis performed by Karapetsas et al. (2012) in an attempt to shed light on the thermocapillary instabilities reported earlier by Sefiane et al. (2008). In the majority of the cases and in most real-life applications, however, spherical evaporating drops are the exception rather than the rule. We address the more complex case of three-dimensional (3D) deformed droplets, and 
with moving contact lines, in the present investigation. To the best of our knowledge, this is the first study of its kind in the literature.

The rest of this paper is organized as follows. Section 2 is devoted to the description of a novel fully-coupled two-phase model based on the diffuse-interface method. The results of an accompanying experimental investigation of evaporating drops in a controlled environment are presented in section 3. These are employed in section 4 to provide the validation of our model. The flow dynamics for two non-spherical pinned drops are examined in section 5 . In addition, the versatility of our model also allows the resolution of the more complex case of drops evaporating with a MCL according to the pure CA mode, which is addressed in section 6 . Finally, the conclusions resulting from this investigation are summarized in section 7 .

\section{Mathematical modelling}

\subsection{Problem statement}

A sketch of the problem is provided in figure 1a. A sessile drop of initial height $\hat{H}_{0}$ and contact radius $\hat{R}_{0}$ resting on a heated substrate evaporates into a non-saturated surrounding gas at atmospheric pressure. The caret decoration denotes dimensional variables. The liquid is a pure substance (water in the experiments) while the gas is modelled as a two-component mixture of variable composition: a non-condensable gas (nitrogen in the experiments) and the liquid's vapour. All three pure substances are regarded as Newtonian fluids. The density, dynamic viscosity, thermal conductivity, and specific heat capacity for the liquid are denoted by $\hat{\rho}_{l}, \hat{\mu}_{l}, \hat{k}_{l}$, and $\hat{c}_{p l}$, respectively. The equivalent physical properties for the non-condensable gas (designated by subscript ' 1 ') and vapour (designated by subscript ' 2 ') are represented by $\hat{\rho}_{g 1}, \hat{\mu}_{g 1}, \hat{k}_{g 1}, \hat{c}_{p g 1}$, and $\hat{\rho}_{g 2}, \hat{\mu}_{g 2}, \hat{k}_{g 2}$, $\hat{c}_{p g 2}$, respectively. The relative amount of the gaseous components is represented by the vapour mass fraction, $\omega$, defined as the mass of vapour per unit mass of gas mixture. The coefficient of binary molecular diffusion is $\hat{D}$, and the specific latent heat is $\Delta \hat{h}_{v}$. The liquid-gas interface is endowed with a surface tension, $\hat{\sigma}$, which decreases monotonically with temperature, $\hat{T}$, according to $\hat{\sigma}=\hat{\sigma}_{0}-\hat{\gamma}\left(\hat{T}-\hat{T}_{a}\right)$; here, $\hat{\sigma}_{0}$ is the surface tension at the reference temperature $\hat{T}_{a}$, and $\hat{\gamma}=-\partial \hat{\sigma} / \partial \hat{T}$ denotes its temperature-dependence coefficient. The substrate is considered to remain isothermal at temperature $\hat{T}_{w}$ while the gas far from the drop is at temperature $\hat{T}_{a}$.

The following scaling is introduced to render the flow variables dimensionless:

$$
\begin{array}{lll}
\hat{\mathbf{x}}=\hat{H}_{0} \mathbf{x}, & \hat{\mathbf{u}}=\hat{U}_{0} \mathbf{u}, & \hat{p}=\hat{\rho}_{l} \hat{U}_{0}^{2} p, \\
\hat{T}=T \Delta \hat{T}+\hat{T}_{a}, & \hat{t}=\frac{\hat{H}_{0}}{\hat{U}_{0}} t, & \hat{\sigma}=\hat{\sigma}_{0} \sigma .
\end{array}
$$

Here, $\mathbf{x}=(x, y, z)$ and $\mathbf{u}=(u, v, w)$ are the coordinate and velocity vectors, $\hat{U}_{0}=$ $\hat{\gamma} \Delta \hat{T} / \hat{\mu}_{l}$ represents the characteristic thermocapillary velocity with $\Delta \hat{T}=\hat{T}_{w}-\hat{T}_{a}, p$ is the pressure, and $t$ denotes the time. The physical properties of the non-condensable gas and vapour are taken into consideration via the following ratios,

$$
\begin{array}{llll}
\Gamma_{\rho}=\frac{\hat{\rho}_{l}}{\hat{\rho}_{g 1}}, & \Gamma_{\mu}=\frac{\hat{\mu}_{l}}{\hat{\mu}_{g 1}}, & \Gamma_{k}=\frac{\hat{k}_{l}}{\hat{k}_{g 1}}, & \Gamma_{c p}=\frac{\hat{c}_{p l}}{\hat{c}_{p g 1}}, \\
\Omega_{\rho}=\frac{\hat{\rho}_{l}}{\hat{\rho}_{g 2}}, & \Omega_{\mu}=\frac{\hat{\mu}_{l}}{\hat{\mu}_{g 2}}, & \Omega_{k}=\frac{\hat{k}_{l}}{\hat{k}_{g 2}}, & \Omega_{c p}=\frac{\hat{c}_{p l}}{\hat{c}_{p g 2}} .
\end{array}
$$

The gas-mixture properties, denoted by $\hat{\rho}_{g}, \hat{\mu}_{g}, \hat{k}_{g}$ and $\hat{c}_{p g}$, depend on the relative amount 


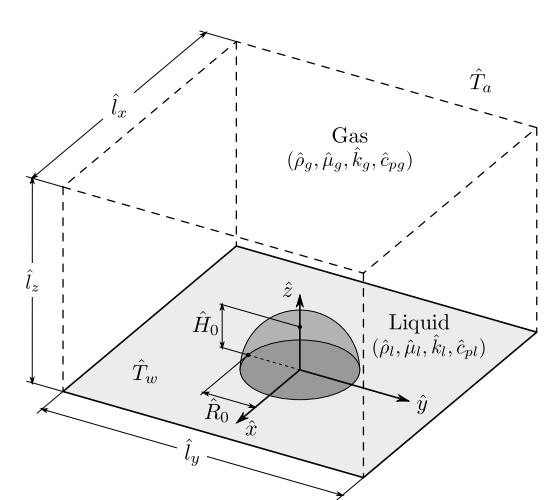

(a)

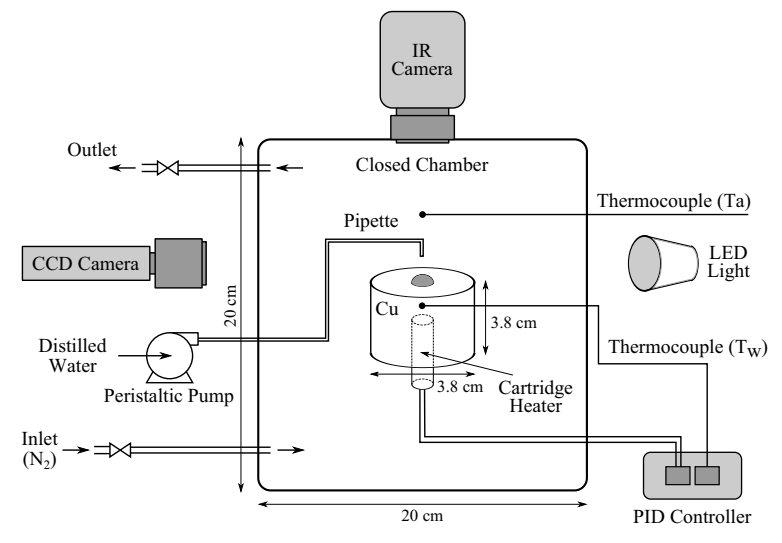

(b)

Figure 1: Schematic of the (a) mathematical model, (b) experimental setup.

of each component as follows,

$$
\begin{aligned}
\rho_{g} & =\left[\Gamma_{\rho}(1-\omega)+\Omega_{\rho} \omega\right]^{-1}, \\
\mu_{g} & =(1-\omega) / \Gamma_{\mu}+\omega / \Omega_{\mu}, \\
k_{g} & =(1-\omega) / \Gamma_{k}+\omega / \Omega_{k}, \\
c_{p g} & =(1-\omega) / \Gamma_{c p}+\omega / \Omega_{c p} .
\end{aligned}
$$

The initial drop geometry $\left(H_{0}, R_{0}\right)$ and the dimensions of the computational domain $\left(L_{x}, L_{y}, L_{z}\right)$ are,

$$
H_{0}=1, \quad R_{0}=\frac{\hat{R}_{0}}{\hat{H}_{0}}, \quad L_{x}=\frac{\hat{l}_{x}}{\hat{H}_{0}}, \quad L_{y}=\frac{\hat{l}_{y}}{\hat{H}_{0}}, \quad L_{z}=\frac{\hat{l}_{z}}{\hat{H}_{0}},
$$

where $\hat{l}_{x}, \hat{l}_{y}$ and $\hat{l}_{z}$ are the width, depth and height of the computational region.

\subsection{Governing equations}

The fully-coupled two-phase dynamics of the drop and surrounding gas are modelled via DNS with a diffuse-interface (DI) method (see Anderson et al. (1998) for a review of this methodology). According to this method, the mathematically-sharp interface is replaced by an interface of finite thickness, which can be prescribed (Jacqmin 1999). We use a DI method based on that developed by Ding et al. (2007) for pure hydrodynamic problems (following Jacqmin (1999), and Badalassi et al. (2003)), but with a number of extensions to address non-isothermal conditions, and phase-change.

DI methods are grounded in two fundamental principles. The first one was given by van der Waals (1979): if the interface between two fluids is regarded as a narrow layer of finite thickness, the volumetric free energy density $\hat{f}$ across it is dependent on the local composition (represented here by the order parameter $c$ ) and their gradients:

$$
\hat{f}=\hat{\varepsilon}^{-1} \hat{\sigma}_{0} \alpha \Psi(c)+\frac{1}{2} \hat{\varepsilon} \hat{\sigma}_{0} \alpha|\hat{\nabla} c|^{2},
$$

where $\hat{\varepsilon}$ is a measure of the interface thickness, $\Psi(c)$ is the bulk energy density with minimum levels corresponding to the fluids' two stable phases, and $\alpha$ is a dimensionless adjustment parameter. In addition, van der Waals (1979) hypothesized that equilibrium interface profiles are those that minimize the total free energy $\hat{\mathscr{F}}=\int_{\Omega} \hat{f} \mathrm{~d} \hat{V}$, where $\Omega$ 
represents the interfacial domain. From the calculus of variations (Jacqmin 1999), these profiles must satisfy,

$$
\hat{\phi}=\hat{\varepsilon}^{-1} \hat{\sigma}_{0} \alpha \Psi^{\prime}(c)+\hat{\varepsilon} \hat{\sigma}_{0} \alpha \hat{\nabla}^{2} c=\text { constant },
$$

where $\hat{\phi}=\partial \hat{\mathscr{F}} / \partial c$ is the chemical potential. The second key element of DI methods was given by Cahn \& Hilliard $(1958,1959)$ and Cahn $(1961)$ who extended the van der Waals' hypothesis to time-dependent situations, postulating that irregular interface profiles recover equilibrium by virtue of diffusion fluxes which are proportional to chemical potential gradients. On these premises, and selecting the liquid volume fraction as an order parameter ( $c=1$ in the liquid, $c=0$ in the gas and $0<c<1$ in the interface region), it can be shown that the spatio-temporal evolution of $c$ is governed by the phase-field, advection-diffusion Cahn-Hilliard equation (Ding et al. 2007):

$$
\frac{\partial c}{\partial t}+\nabla \cdot(c \mathbf{u})-\frac{1}{P e} \nabla \cdot(M \nabla \phi)=0
$$

where $P e=\hat{U}_{0} \hat{H}_{0} /\left(\hat{M}_{0} \hat{\phi}_{0}\right)$ is the Péclet number, $M=c(1-c)$ is a diffusive coefficient referred to as "mobility", $\phi=\varepsilon^{-1}\left[\Psi^{\prime}(c)-\varepsilon^{2} \nabla^{2} c\right]$ is the chemical potential, and $\Psi(c)=$ $\frac{1}{4} c^{2}(1-c)^{2}$. With appropriate boundary conditions, equation 2.7 conforms to a massconservative interface-capturing method, in which the the interface profile is maintained regular by means of the diffusive term. Some recent examples wherein this equation has been used include droplet spreading (Ding \& Spelt 2007a), droplet motion under shear flow (Ding \& Spelt 2008; Ding et al. 2010), and two-layer flows (Valluri et al. 2010).

For an evaporating drop, the total mass of liquid and vapour is globally conserved, but the mass of liquid is not; this decreases in time due to the liquid-to-gas phase change. Thus, accounting for interface mass transfer requires adding an interface sink/source term in equation 2.7, which leads to the phase-change version of the Cahn-Hilliard equation:

$$
\frac{\partial c}{\partial t}+\nabla \cdot(c \mathbf{u})-\frac{1}{P e} \nabla \cdot(M \nabla \phi)=-S,
$$

where $S$ is the volumetric interface mass-transfer rate (only computed in the interface region). The negative sign on the RHS of 2.8 results from our sign convention, namely $S>0(S<0)$ for evaporation (condensation). Following the traditional assumption that the gas at the interface is (quasi) saturated with vapour (Picknett \& Bexon 1977; Deegan et al. 1997; Hu \& Larson 2002; Cazabat \& Guena 2010), which is valid for slow evaporation cases as the one considered here, $S$ is calculated as follows,

$$
S=\frac{(1-c) \rho_{g}}{t_{s}}\left[\frac{p_{s}}{p_{s}+\frac{1}{\mathscr{M}}\left(\frac{1}{\mathscr{P}}-p_{s}\right)}-\omega\right],
$$

where $p_{s}$ denotes the saturation vapour pressure, $\mathscr{M}=\hat{M}_{g 2} / \hat{M}_{g 1}$ is the vapour-gas molar weight ratio, $\mathscr{P}=\hat{\rho}_{l} \hat{U}_{0}^{2} / \hat{p}_{r}$, is a dimensionless group including the absolute pressure $\hat{p}_{r}$, and $t_{s}$ is a measure of interface mass-transfer rate small enough to maintain saturation condition at the interface. The first term within the brackets represents the saturation vapour mass fraction calculated using Raoult's law and Dalton's law of partial pressure, i.e. it is assumed that the gaseous components form an ideal mixture. The detailed justification of this expression is given in Sáenz et al. (2014). The saturation pressure dependence on $T$ is approximated with a standard Antoine expression, $\log _{10}\left(\hat{p}_{s}\right)=\hat{A}-$ $\hat{B} /(\hat{C}+\hat{T})$, where $\hat{A}, \hat{B}$ and $\hat{C}$ are the empirical coefficients.

The overall mass balance is completed with the continuity equation, while the conser- 
vation of momentum is governed by the Navier-Stokes equations:

$$
\begin{gathered}
\nabla \cdot \mathbf{u}=-S\left(1-\Omega_{\rho}\right), \\
\frac{\partial(\rho \mathbf{u})}{\partial t}+\nabla \cdot(\rho \mathbf{u u})=-\nabla p+\frac{1}{R e} \nabla \cdot\left[\mu\left(\nabla \mathbf{u}+\nabla \mathbf{u}^{T}\right)\right]+\frac{1}{W e} \phi \nabla c-\frac{1}{R e}\left(\nabla_{\mathrm{s}} T\right) \delta,
\end{gathered}
$$

where $\rho=c+\rho_{g}(1-c)$ and $\mu=c+\mu_{g}(1-c)$ are the one-fluid form of the density and viscosity respectively, $R e=\hat{\rho}_{l} \hat{U}_{0} \hat{H}_{0} / \hat{\mu}_{l}$ is the Reynolds number, $W e=\hat{\rho}_{l} \hat{U}_{0}^{2} \hat{H}_{0} /\left(6 \sqrt{2} \hat{\sigma}_{0}\right)$ denotes the Weber number, $\phi \nabla c$ is the DI interpretation of the mean surface tension stress enforcing (Jacqmin 1999; Ding et al. 2007), and $\left(\nabla_{\mathrm{s}} T\right) \delta$ models the thermocapillarity effect with $\nabla_{\mathrm{s}}=\nabla-\mathbf{n}(\mathbf{n} \cdot \nabla)$ being the gradient tangent to the interface, $\mathbf{n}=\nabla c /|\nabla c|$ the inwards unit vector normal to the interface, and $\delta=|\nabla c|$. It should be noted that several authors (Jacqmin 2000; Ding \& Spelt 2007a; Yue et al. 2010) have already shown that the results from this DI approach converge to the sharp-interface limit in the limit of zero interfacial thickness.

The conservation of energy in both phases is computed via

$$
\frac{\partial\left(\rho c_{p} T\right)}{\partial t}+\nabla \cdot\left(\rho c_{p} T \mathbf{u}\right)=\frac{1}{\operatorname{RePr}} \nabla \cdot(k \nabla T)-\frac{1}{J a} S .
$$

Here, $k=c+k_{g}(1-c)$ and $c_{p}=\left(c+\rho_{g} c_{p g}(1-c)\right) /\left(c+\rho_{g}(1-c)\right)$ are the one-fluid form thermal conductivity and specific heat capacity, and $\operatorname{Pr}=\hat{\mu}_{l} \hat{c}_{p l} / \hat{k}_{l}$ and $J a=\hat{c}_{p l} \Delta \hat{T} / \Delta \hat{h}_{v}$ are the Prandtl and Jakob numbers, respectively. Finally, the vapour mass fraction, $\omega$, is governed by a general advection-diffusion transport equation only solved in gas phase:

$$
\frac{\partial\left[\rho_{g}(1-c) \omega\right]}{\partial t}+\nabla \cdot\left[\rho_{g}(1-c) \omega \mathbf{u}\right]=\frac{1}{R e S c} \nabla \cdot\left[\rho_{g}(1-c) \nabla \omega\right]+S,
$$

where $S c=\hat{\mu}_{l} /\left(\hat{\rho}_{l} \hat{D}\right)$ is the Schmidt number.

\subsection{Boundary and initial conditions}

The boundary conditions are selected to mimic the experiments presented in section 3 . The lower boundary $(z=0)$ is modelled as an isothermal $(T=1)$, no-slip $(\mathbf{u}=0)$ solid substrate with nonpenetration of mass $\left(\mathbf{n}_{z} \cdot \nabla \omega=0\right)$. Here, $\mathbf{n}_{x}, \mathbf{n}_{y}$, and $\mathbf{n}_{z}$ denote the unit vectors in the principal directions. The Cahn-Hilliard equation requires the Neumann boundary condition $\mathbf{n}_{z} \cdot \nabla \phi=0$ to guarantee the volume conservation of each fluid (Ding \& Spelt 2007b). Another condition on $c$ is necessary; this being a Dirichlet or a Neumann condition in pinned, or moving contact line configurations, respectively. A number of models have been proposed to solve the dynamics of moving contact lines, e.g. Qian et al. (2006), Ding \& Spelt (2007b), Carlson et al. (2009) and Yue et al. (2010). The approach suggested by Ding \& Spelt $(2007 b)$ is adopted here. The contact angle is, therefore, prescribed by virtue of the boundary condition:

$$
\mathbf{n}_{\mathrm{w}} \cdot \nabla c=-\cos \theta_{s}\left|\mathbf{t}_{\mathrm{w}} \cdot \nabla c\right| / \sin \theta_{s}
$$

where $\theta_{s}$ is the microscale contact angle, and $\mathbf{n}_{\mathrm{w}}$ and $\mathbf{t}_{\mathrm{w}}$ are the unit vectors normal and tangential to the solid wall, respectively (Ding \& Spelt 2007b). It is important to emphasize that prescribing a slip velocity is unnecessary, given that the slow contact line motion is solved by virtue of finite diffusion (Sui et al. 2014). The temperature of the upper boundary $\left(z=L_{z}\right)$ is set to the ambient temperature as recorded by the secondary thermocouple $(T=0)$ while Neumann boundary conditions $\left(\mathbf{n}_{x} \cdot \nabla T=0\right.$, and $\left.\mathbf{n}_{y} \cdot \nabla T=0\right)$ are used at the vertical walls of the computational domain $\left(x= \pm L_{x} / 2\right.$ and $y= \pm L_{y} / 2$ ). 
In the experimental setup, the size of chamber was intentionally designed to be very large in comparison to that of the drop so this would evaporate into a much larger volume of pure $\mathrm{N}_{2}$ (dry gas). An approximately infinite amount of $\mathrm{N}_{2}$ surrounding the drop makes it is reasonable to assume that the phase-change does not result in a noteworthy increment in the bulk humidity. Thus, the zero Dirichlet boundary condition is imposed in the vertical and top boundaries $\left(\omega=0\right.$ at $x= \pm L_{x} / 2, y= \pm L_{y} / 2$, and $z=L_{z}$ ). Provided that the computational domain is large enough, the vapour distribution around the drop becomes independent of the far-field boundary conditions. For the same reason, it is considered that the flow far away from the drop is at rest $\left(\mathbf{u}=0\right.$ at $x= \pm L_{x} / 2$, $y= \pm L_{y} / 2$, and $z=L_{z}$ ). Finally, the fact that the species equation is only solved in the gas phase requires the implicit imposition of the no-penetration condition $(\mathbf{n} \cdot \nabla \omega=0$, and $\mathbf{n} \cdot \mathbf{u}=0$ ) along the species-field boundary in the liquid-gas interface (contour $c=0.9$ ).

Initial conditions are also prescribed. At $t=0$, the drop is a spherical cap of height $H_{0}=1$ and base radius $R_{0}$; the two-phase flow is at rest $(\mathbf{u}=0)$, and the gas-mixture is completely dry $(\omega=0)$. The initial pressures in the gas, and within the drop are set to zero, and the Laplace pressure, respectively. In dimensionless form, the latter becomes $\Delta p=1 /\left(3 \sqrt{2} W e R_{s}\right)$ with $R_{s}=\left(H_{0}^{2}+R_{0}^{2}\right) /\left(2 H_{0}\right)$. From the experiments, it is known that the chamber was allowed to reach thermal equilibrium before the drop was deposited onto the substrate, and that the liquid dosed through the pipette was pumped from a reservoir located outside the chamber and was, therefore, at ambient temperature. These two considerations led us to select the initial drop temperature as $T=0$, and the initial temperature of the gas as a linear distribution from the substrate to the ambient temperature, i.e. $T=\left(L_{z}-z\right) / L_{z}$. Both conditions are simultaneously met with $T=(1-c)\left(L_{z}-z\right) / L_{z}$.

\subsection{Numerical method}

The governing equations 2.8, 2.10-2.13 coupled with the volumetric interface masstransfer rate calculated with equation 2.9, constitute a system of seven Partial Differential Equations (PDEs) with seven unknowns $(c, p, u, v, w, T$ and $\omega)$. This system of equations is simultaneously solved (DNS) with a finite-volume (FV) discretization of the physical domain by means of a staggered Marker-And-Cell (MAC) grid: scalar variables $(c, p, T$, $\omega)$ are stored in the cell centres while the velocity components $(u, v, w)$ are defined at the control-volume faces. Spatial derivatives are approximated by a centered scheme. Mesh refinement tests are carried out to ensure that the results are grid-independent.

The Cahn-Hilliard equation is solved numerically using the implicit-explicit strategy proposed by Badalassi et al. (2003) with a second order semi-backward difference formula (SBDF) scheme (see Ascher et al. (1995)). The convective term is explicitly computed approximating the fluxes at cell faces with an upwind (referred to the flow direction) fifthorder weighted essentially non-oscillatory (WENO) scheme (Liu et al. 1994) to increase the code's stability to the shock-like nature of the $c$ profile across the interface (Ding et al. 2007). The resolution of the velocity and pressure fields is achieved by a standard projection method wherein the intermediate velocity (without considering $p$ ) is found with the second-order Crank-Nicolson Adams-Bashforth (CNAB) scheme to approximate the diffusive (implicitly) and advective (explicitly) terms, respectively. The pressure is then added to update the velocity to its final value ensuring that the continuity condition is satisfied. The energy and species fields are advanced in time with similar semi-implicit CNAB techniques.

The solution strategy requires knowing the value of all the variables at the current timestep $(n)$ as well as $c$ at the previous time-step $(n-1)$. The computation then proceeds by updating the volume fraction via 2.8 with $\mathbf{u}$ and $S$ evaluated at $(n)$. Equations 2.13 and 
2.12 are then solved sequentially. This order results naturally from the fact that the former involves $c^{n+1}$ (to evaluate $\rho_{g}$ ) while the latter additionally requires $\omega^{n+1}$ (to evaluate $\rho, k$ and $c_{p}$ ). Finally, with $c^{n+1}, \omega^{n+1}$ and $T^{n+1}$, the velocity is updated solving equations 2.10 and 2.11 with the surface tension and physical properties evaluated at $(n+1 / 2)$ as recommended by Ding et al. (2007). Following the study conducted by Ding et al. (2007), the Péclet number is set to $P e=1 / \varepsilon^{2}$, choosing $\varepsilon$ to be proportional to the grid resolution, i.e. $\varepsilon=0.5 \min (\Delta x, \Delta y, \Delta z)$. This configuration leads to an interface thickness, i.e. distance between contours of $c=0.1$ and 0.9 , of around three times the minimum grid spacing. The relevant timescales in the system are the viscous, conductive, convective, molecular diffusivity and evaporative timescales. The marching time is selected to be smaller than all of them, i.e. $\Delta t<\min \left(\Delta \hat{x}^{2} / \hat{\nu}_{l}, \Delta \hat{x}^{2} / \hat{\alpha}_{l}, \Delta \hat{x} / \hat{u}_{0}, \Delta \hat{x}^{2} / \hat{D}, \hat{\rho}_{g} / \hat{S}_{\max }\right) / \hat{t}_{0}$. This temporal scale is also used for $t_{s}$. The numerical method is coded in Fortran 90 for implementation on a shared-memory architecture, using Open Multi-Processing (OpenMP). The code is run on the supercomputer ARCHER (Cray XC30 Supercomputer) using a $2.7 \mathrm{GHz}$, 12-core E5-2697 v2 (Ivy Bridge) series processor for a typical simulation.

\section{Experiments}

\subsection{Experimental apparatus}

An experimental apparatus was designed to measure the profile of evaporating drops using optical techniques. A schematic of the setup is presented in figure 1b. Very small drops $\left(\hat{V}_{0} \sim 10 \mu \mathrm{L}\right)$ of distilled water were gently deposited on a heated substrate, allowed to evaporate, and simultaneously recorded from the side by a CCD camera and from the top by an infra-red (IR) camera. The substrate was a copper cylinder of dimensions $3.8 \mathrm{~cm}$ diameter and $3.8 \mathrm{~cm}$ length. This was maintained at constant temperature, $\hat{T}_{w}$, by means of a cartridge heater inserted within the cylinder from below (Omega CSS-10120/120V, $20 \mathrm{~W}, 2.54 \mathrm{~cm}$ length and $0.64 \mathrm{~cm}$ diameter), a PID controller $\pm 0.2^{\circ} \mathrm{C}$ (Omega CN77544A2) and a K-type thermocouple located in a small hole $6 \mathrm{~mm}$ below the substrate's upper surface. Copper and the cylinder dimensions were chosen to provide a substrate with very high thermal conductivity as well as very large thermal mass so that the surface remains isothermal throughout the evaporation process. To ensure a smooth finish, the copper substrate was progressively ground, and polished (with $0.05 \mu \mathrm{m}$ colloidal alumina suspension in the final stage). The emissivity for water and polished copper are $\epsilon=0.96$ and 0.03 , respectively, which results in high-contrast IR images wherein the interface temperature and contact line were sharply defined.

The size of the drops was controlled via the pipette tip size, tube diameter, and peristaltic pump speed. In order to avoid imprecision in assessing the composition (physical properties), and relative humidity (driving force) of the air surrounding the drop, the substrate and dosing mechanism were placed within a closed, acrylic cubic chamber (dimensions $20 \times 20 \times 20 \mathrm{~cm}$ and $0.54 \mathrm{~cm}$ wall thickness) maintained at atmospheric pressure. A system of valves was attached to this chamber to allow the replacement of the ambient air by pure nitrogen $\left(\mathrm{N}_{2}\right)$. The use of a closed chamber also guaranteed that the evaporation process was not distorted by externally-induced convection currents and that the IR readings were not contaminated by radiation from the surroundings, since acrylic is opaque in the spectrum range of our IR camera. Inner reflections were also minimized by painting the interior of the chamber with black paint, except for two small gaps on opposite sides to allow the CCD recording and back-lighting of the drop, respectively.

The drop profiles were captured with a CCD camera $(900 \times 600$ pixels, $9 \mu \mathrm{m} /$ pixel $)$, capable of recording up to 30 frames per second, connected to a video-digitizer board 
(frame grabber). LED back-lighting was employed to improve the video's contrast without raising the temperature. The recorded side images were later post-processed using the Droplet Shape Analyser from Krüss (DSA v1.9, Krüss GmbH, Hamburgh, Germany) in order to obtain the instantaneous height $\hat{H}$, contact angle $\theta$, base radius $\hat{R}$ and volume $\hat{V}$ throughout the evaporation process. Simultaneously, a midwave IR camera mounted directly above the substrate and facing vertically downwards onto the drop (FLIR Silver SC55600, spectrum range $3-5 \mu \mathrm{m}, 640 \times 512$ pixels, $100 \mathrm{fps})$ was used to record the interface temperature field and contact-line dynamics.

A cylindrical black body, as described in Kim et al. (2012), was used to calibrate the IR readings. Before every experimental run, $\mathrm{N}_{2}$ was blown through the chamber for a sufficiently long period of time to make sure that this was the only gas present within the test cell. The inlet and outlet valves were then closed and the system was allowed to reach equilibrium. Steady state was recognized by means of a secondary thermocouple located in the gas, $3.5 \mathrm{~cm}$ above the heated substrate, which also provided the real 'bulk' or 'ambient' temperature, $\hat{T}_{a}$. Only after the system reached equilibrium, detected when $\hat{T}_{a}$ became steady, was a single drop released onto the heated surface and the recording process started. This procedure was repeated for every drop in this investigation to ensure consistency.

\subsection{Results}

The evaporation of water drops on a heated copper substrate is presented for $\hat{T}_{w}=40,55$, and $70^{\circ} \mathrm{C}$. The average initial volume and contact angle were $\hat{V}_{0}=10.4 \mu \mathrm{L} \pm 4.7 \%$ and $\theta_{0}=84^{\circ} \pm 1.6 \%$, respectively. This led to drops whose initial height and base radius were $\hat{H}_{0}=1.55 \mathrm{~mm} \pm 1.5 \%$ and $\hat{R}_{0}=1.86 \mathrm{~mm} \pm 2.4 \%$, respectively. Drops outside this range of parameters were dismissed. For each value of $\hat{T}_{w}$, the experiment was repeated at least five times to ensure reproducibility. Under terrestrial gravity, the capillary length $\hat{\lambda}_{c}=\sqrt{\hat{\sigma}_{0} / \hat{g} \hat{\rho}_{l}}$ for water is $2.73 \mathrm{~mm}$. Since $\hat{H}_{0}$ is well below $\hat{\lambda}_{c}$, it may be concluded that capillary stresses are dominant over gravity forces and, therefore, the drops take the shape of spherical caps. The same conclusion is drawn by examining the static Bond number $B o=\hat{\rho}_{l} \hat{g} \hat{H}_{0}^{2} / \hat{\sigma}_{0}=0.3$, which, given its small value, denotes the dominance of surface tension. The analysis of the drop profiles recorded by the CCD camera allowed us to estimate quantitatively the effect of gravity in a maximum $\theta$ deviation of $4^{\circ}(t=0)$ between the typical experimental drop $\left(\theta_{0}=84^{\circ}\right)$ and a perfect spherical cap with the same $V$ and $R\left(\theta_{0}=80^{\circ}\right)$. Note that this deviation is further minimized in time given that $H$ decreases due to evaporation.

Independently of $\hat{T}_{w}$, the typical evaporation process comprises two well-defined stages, as shown in figure 2. Initially, and for most of their lifetime, all drops evaporate according to the CR mode. Both CCD and IR recordings show that the contact line remains circular in its original position while $\hat{H}$ and $\theta$ progressively decrease (see figure 2a-c). This first stage is followed by a second phase wherein the drop evaporates according to the SS mode. Once the contact angle reaches certain critical value $\theta=\theta_{c}$, the drop experiences a sudden readjustment process to regain equilibrium in which its contact surface undergoes a significant reduction (see figure 2c and d). Normally, a segment of the triple line depins (bottom-right section in figure $2 \mathrm{~d}$ ) while the rest remains anchored to its original location (top-left section in figure $2 \mathrm{~d}$ ). The location of the pinned section appears to be random and is related to microscopic surface heterogeneities in the surface roughness. This stick-slip process usually repeats itself several times before the drop dries out. Once the depinning of the contact line takes place for the first time, interface sphericity is lost for all the cases studied. These observations are in line with the previous 


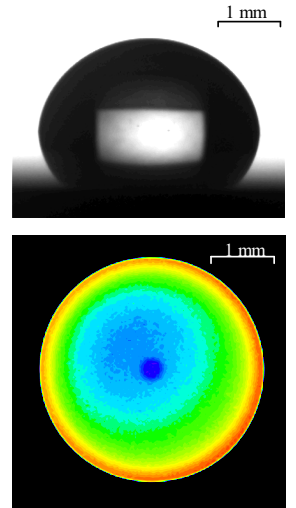

(a) $t / t_{\text {tot }}=0$
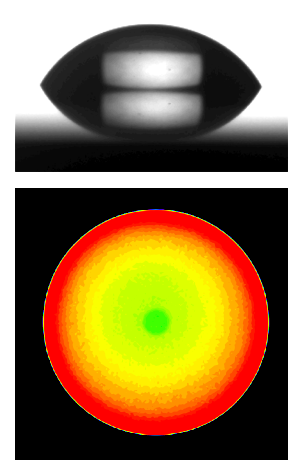

(b) $t / t_{\text {tot }}=0.4$
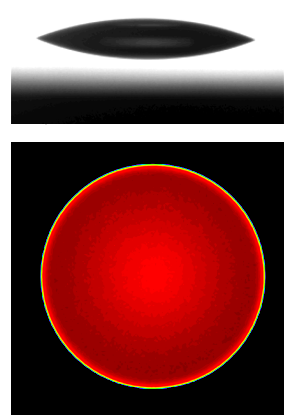

(c) $t / t_{\text {tot }}=0.8$
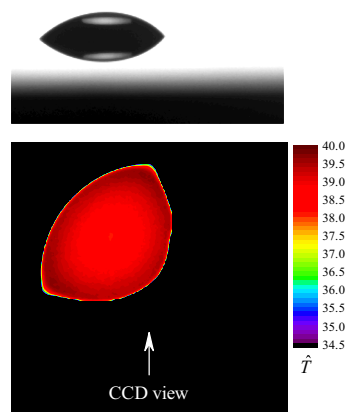

(d) $t / t_{\text {tot }}=0.85$

Figure 2: Evaporation of a water drop into $\mathrm{N}_{2}$ simultaneously recorded with a CCD camera from the side (top row) and with an IR camera from the top (bottom row) with $\hat{T}_{w}=40^{\circ} \mathrm{C}$ and $\Delta \hat{T}=14.6^{\circ} \mathrm{C}$. The typical evaporation time is $\hat{t}_{\text {tot }}=835 \mathrm{~s}$

studies available in the literature, e.g. Crafton \& Black (2004), Shanahan et al. (2011), Orejon et al. (2011) or Nguyen et al. (2012).

The IR recordings reveal that the interface temperature follows a concentric distribution, from a value close to $\hat{T}_{w}$ near the contact line to a colder $\hat{T}$ at the apex (see figure 2 bottom). The dot observed at the centre of the interface is the reflection of the IR camera. No thermal motion in either the azimuthal or the radial direction is observed. This is in agreement with the work by Sefiane et al. (2008) who discovered significant temperature instabilities in drops of ethanol, methanol, and FC-72 but not water. The difference between the interface and substrate temperature is maximum at the start of the experiment. As the drop evaporates and $\hat{H}$ decreases, the radial temperature gradient becomes less pronounced until the interface temperature eventually increases to almost reach $\hat{T}_{w}$ (figure 2c). Hence, the Marangoni flow within the drop weakens as the drop becomes thinner, given that the radial temperature gradient is the driving force. The readings of the secondary thermocouple, located above the drop, indicate that ambient temperature $\hat{T}_{a}$ is not constant as is often assumed. The temperature $\hat{T}_{a}$ rises with $\hat{T}_{w}$, which means that the effective temperature difference in the vertical direction, $\Delta \hat{T}=\hat{T}_{w}-\hat{T}_{a}$, is, in general, smaller than when this is evaluated with a fixed room temperature. In our experiments, $\hat{T}_{a}=25.4^{\circ} \mathrm{C}\left(\Delta \hat{T}=14.6{ }^{\circ} \mathrm{C}\right), 26.3^{\circ} \mathrm{C}\left(28.7^{\circ} \mathrm{C}\right)$, and $30.2^{\circ} \mathrm{C}\left(39.8^{\circ} \mathrm{C}\right)$ for $\hat{T}_{w}=40,55$, and $70^{\circ} \mathrm{C}$, respectively. These readings are used in the accompanying simulations to model the surrounding gas more realistically.

The evolution in time of $\theta, \hat{H}, \hat{R}$, and $\hat{V}$ over the range of $\hat{T}_{w}$ is depicted in figure 3 . The evaporation time required for complete dry-out decreases with increasing $\hat{T}_{w}: \hat{t}_{\text {tot }}=835$, 354 , and $180 \mathrm{~s}$ for $\hat{T}_{w}=40,55$, and $70^{\circ} \mathrm{C}$, respectively, with a standard deviation $<7 \%$ in all cases. The initial (equilibrium) contact angle $\theta_{0}=84 \pm 1.6^{\circ}$ seems weakly affected by $\hat{T}_{w}$; slightly larger degrees of hydrophobicity are found with colder substrates, which is due to a decrease of surface tension with temperature. A similar but significantly more pronounced variation in $\theta_{0}$ due to $\hat{T}_{w}$ was observed by Mollaret et al. (2004) with water on aluminium. Figure $3 \mathrm{a}$ shows that $\theta$ decreases at a progressively-increasing rate until the three-dimensional motion of the drop begins to recover equilibrium at a new position. Simultaneously, $\hat{H}$ registers an equivalent type of nonlinear evolution, see figure $3 \mathrm{~b}$. At higher $\hat{T}_{w}$, the transition from the initial CR to the final SS mode is very well defined. 
(a)

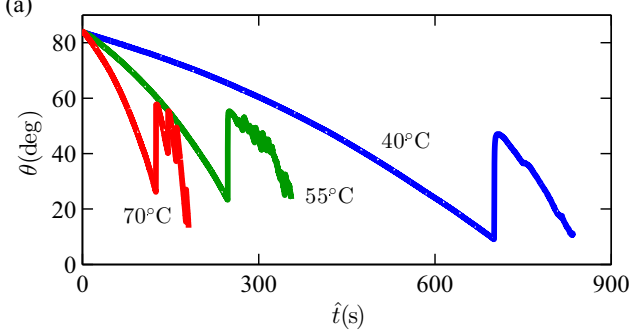

(c)

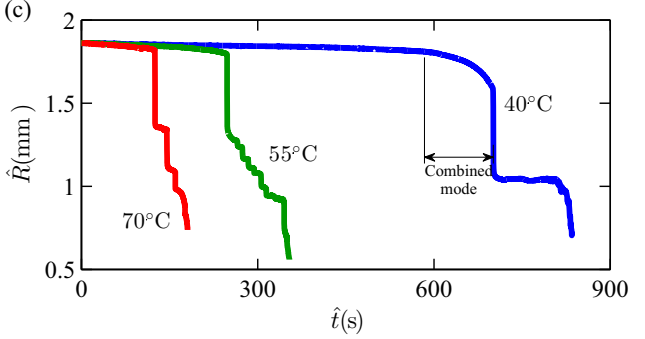

(b)

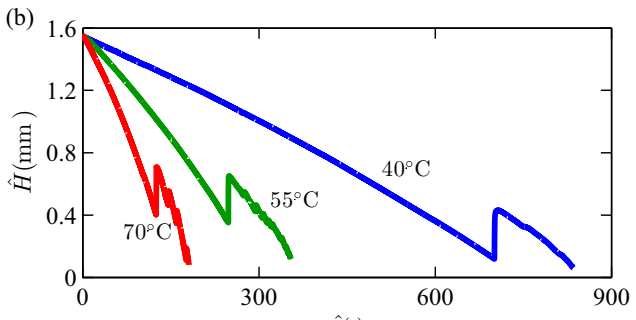

$\hat{t}(\mathrm{~s})$

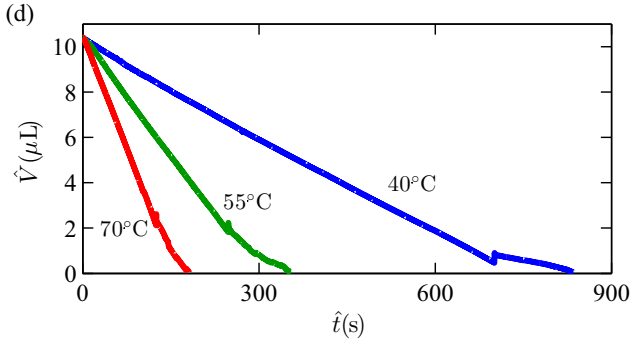

Figure 3: Instantaneous evolution of the (a) contact angle $\theta$, (b) maximum height $\hat{H}$, (c) contact radius $\hat{R}$, and (d) volume $\hat{V}$ for a water drop evaporating into $\mathrm{N}_{2}$ placed on a heated substrate at various temperatures $\hat{T}_{w}$.

This occurs at $\theta_{c}=22 \pm 3^{\circ}(\hat{t}=248 \mathrm{~s})$ and $26 \pm 3^{\circ}(125 \mathrm{~s})$ for $\hat{T}_{w}=55$ and $70^{\circ} \mathrm{C}$, respectively, which compares well with the $20-40^{\circ}$ range reported by Crafton \& Black (2004) for $60^{\circ} \mathrm{C} \leqslant \hat{T}_{w} \leqslant 95^{\circ} \mathrm{C}$. However, when the substrate is colder, $\hat{T}_{w}=40^{\circ} \mathrm{C}$, the drop goes through a transition stage wherein the evaporation mechanism is actually a combined mode. This occurs just before the first contact-line jump at $\theta_{c}=10 \pm 2^{\circ}$ $(\hat{t}=701 \mathrm{~s})$. Figure $3 \mathrm{a}$ and c illustrate how both $\theta$ and $\hat{R}$ decrease simultaneously between $\hat{t} \sim 580-701 \mathrm{~s}$, which suggests that this intermediate stage fits neither the CR nor the CA pure modes of evaporation. Thus, the transition from the CR to SS mode is not instantaneous as for $\hat{T}_{w}=55$ or $70^{\circ} \mathrm{C}$. This behaviour was observed in all five runs.

While the drop is pinned, the instantaneous $\hat{V}$ is essentially linear for any $\hat{T}_{w}$, see figure 3 d. Hence, the resulting evaporation rate, $\hat{m}(\hat{t})=-\mathrm{d} \hat{V} / \mathrm{d} \hat{t}$, is almost constant in the CR mode, which is in agreement with previous works, e.g. Picknett \& Bexon (1977); BourgèsMonnier \& Shanahan (1995); Mollaret et al. (2004); Cazabat \& Guena (2010); Shanahan et al. (2011). During this phase, the experiments show that the average evaporation rate is $\hat{m}(\hat{t})=0.0135 \pm 7.5 \times 10^{-4}, 0.0340 \pm 1.4 \times 10^{-3}$, and $0.0698 \pm 1.8 \times 10^{-3} \mu \mathrm{L} \mathrm{s}^{-1}$ for $\hat{T}_{w}=40,55$, and $70^{\circ} \mathrm{C}$, respectively. Deviations from the linear behaviour of $\hat{m}(\hat{t})$ are noted with $\hat{T}_{w}=40^{\circ} \mathrm{C}$ when the drop is subjected to the combined evaporation mode, $\hat{t} \sim 580-701 \mathrm{~s}$. In all cases, after the contact-line readjustment, $\hat{m}(\hat{t})$ is lower than that prior to the drop depinning because the contact line length and the liquid-gas interfacial area have decreased.

\section{Axisymmetrical drops - Model validation}

We start the modelling work by considering axisymmetrical pinned drops, i.e. CR mode. This is consistent with the evaporation process observed during most of the drop's lifetime $(70 \%)$. In all cases investigated experimentally (figure 3 ), the maximum deviation $\left(\hat{R}_{0}-\hat{R}\right) / \hat{R}_{0}$ after this time is $\leqslant 3 \%$ and, therefore, assumed to be negligible to maintain the mathematical problem as simple as possible. The initial height and base radius of 
Table 1: Physical properties of distilled water (liquid and vapour) and nitrogen gas at $\hat{T}=25{ }^{\circ} \mathrm{C}$. The empirical coefficient for the Antoine equation $\log _{10}\left(\hat{p}_{s}\right)=\hat{A}-\hat{B} /(\hat{C}+\hat{T})$, with $\hat{p}_{s}$ in $(\mathrm{Pa})$ and $\hat{T}$ in $\left({ }^{\circ} \mathrm{C}\right)$, are $\hat{A}=8.0557, \hat{B}=1723.6$, and $\hat{C}=233.08$. ${ }^{\dagger}$ Updated for $\hat{T}=40^{\circ} \mathrm{C}$.

\begin{tabular}{llccc} 
& & Water (Liquid) & Water (Vapour) & $\mathrm{N}_{2}$ \\
\hline$\hat{\rho}$ & $\left(\mathrm{kg} \mathrm{m}^{-3}\right)$ & 997 & 0.736 & 1.14 \\
$\hat{\mu}$ & $\left(\mathrm{Pa} \mathrm{s}^{-1} \mathrm{~K}^{-1}\right)$ & $8.90 \times 10^{-4}$ & $9.97 \times 10^{-6}$ & $1.73 \times 10^{-5}$ \\
$\hat{k}$ & $\left(\mathrm{~W} \mathrm{~m}^{-1} \mathrm{~K}^{-1}\right)$ & 4180 & $1.86 \times 10^{-2}$ & $2.57 \times 10^{-2}$ \\
$\hat{c}$ & $\left(\mathrm{~J} \mathrm{~kg}^{-1}\right)$ & 1864 & 1040 \\
$\hat{M}$ & $\left(\mathrm{~g} \mathrm{~mol}^{-1}\right)$ & 18.02 & & 28.01 \\
$\hat{\sigma}$ & $\left(\mathrm{N} \mathrm{m}^{-1}\right)$ & $7.27 \times 10^{-2}$ & & \\
$\hat{\gamma}$ & $\left(\mathrm{N} \mathrm{m}^{-1} \mathrm{~K}^{-1}\right)$ & $1.71 \times 10^{-4}$ & & \\
$\Delta \hat{h}_{v}$ & $\left(\mathrm{~J} \mathrm{~kg}^{-1}\right)$ & $2.40 \times 10^{6}$ & & \\
$\hat{D}$ & $\left(\mathrm{~m}^{2} \mathrm{~s}^{-1}\right)$ & $2.84 \times 10^{-5 \dagger}$ & &
\end{tabular}

the drop for the simulations are those from the experiments, i.e. $\hat{H}_{0}=1.55 \mathrm{~mm}\left(H_{0}=1\right)$ and $\hat{R}_{0}=1.86 \mathrm{~mm}\left(R_{0}=1.2\right)$, respectively. In what follows, the discussion is restricted to drops at moderate heating, i.e. $\hat{T}_{w}=40^{\circ} \mathrm{C}$ and $\Delta \hat{T}=14.6^{\circ} \mathrm{C}$, for the most part.

The physical properties of distilled water and nitrogen are listed in table 1 . In the range considered, the variations of these properties due to $\hat{T}$ are negligible for the liquid $(<3 \%)$ and gases $(<5 \%)$ with the exception of the coefficient of binary mass diffusion $\hat{D}$ $\left(13 \%\right.$ higher at $\left.40^{\circ} \mathrm{C}\right)$ and liquid viscosity $\hat{\mu}_{l}(27 \%$ lower $)$. For the former, we employ the corrected value given that the evaporation rate $\hat{m}(\hat{t})$ is very sensitive to it. For the latter, our tests show that reducing $\hat{\mu}_{l}$ increases exponentially the computational difficulty of the problem without revealing any significant changes in the two-phase dynamics of the flow for the purpose of this investigation. Note that $\hat{\mu}_{l}^{2}$ appears in the denominator of $R e$. This also provides a rationale for focusing on moderate heating. The governing dimensionless groups become $R e=4830, W e=19.4, P r=6.1, J a=0.025, S c=0.032, \Gamma_{\rho}=870$, $\Gamma_{\mu}=50, \Gamma_{k}=24, \Gamma_{c p}=4, \Omega_{\rho}=1350, \Omega_{\mu}=90, \Omega_{k}=33, \Omega_{c p}=2.2, \mathscr{M}=6.433 \times 10^{-1}$, and $\mathscr{P}=7.632 \times 10^{-2}$. These dimensionless numbers are maintained for the simulation of non-spherical drops presented in section 5 as well as for the drops evaporating with a moving contact line examined in section 6 .

When selecting the domain size, it must be noted that $m(t)$ decreases asymptotically for increasing size. We conducted tests with $L_{r}=L_{z}=10 R_{0}, 20 R_{0}, 50 R_{0}$, and $100 R_{0}$, where $L_{r}$ and $L_{z}$ are the 2D domain dimensions in the radial and axial directions, respectively. Taking the previous smaller domain as reference, $m(t)$ decreases $3.7 \%, 1.5 \%$, and $\sim 0 \%$ for $20 R_{0}, 50 R_{0}$, and $100 R_{0}$, respectively. The $m(t)$ variation from $20 R_{0}$ to $50 R_{0}(1.5 \%)$ is acceptable and, therefore, we selected $L_{r}=L_{z}=20 R_{0}$ for our simulations in order to minimize the computational cost. Thus, the domain size is much larger than the drop and the evaporation process becomes independent of the vertical and upper boundary conditions. Note that $\mathrm{Hu} \&$ Larson (2002) arrived at the same conclusion in terms of the domain size for the non-heated case. The domain is discretised by means of a structured mesh of $250 \times 250$ elements with finer and uniform resolution around the drop, i.e. $125 \times 125$ for $r, z<1.5 R_{0}$, and geometrically-decreasing resolution for increasing $r$ and $z$; common ratio 1.0321. This grid is chosen based on the results from mesh-dependency 
(a)

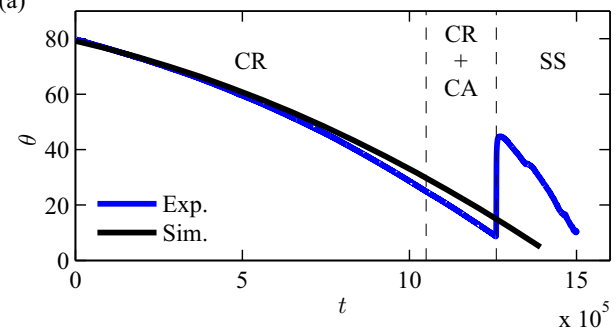

(c)

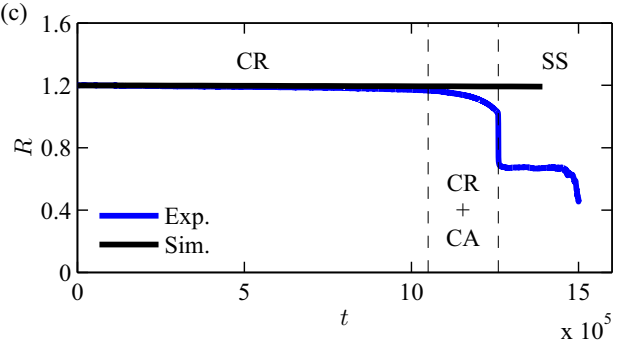

(b)

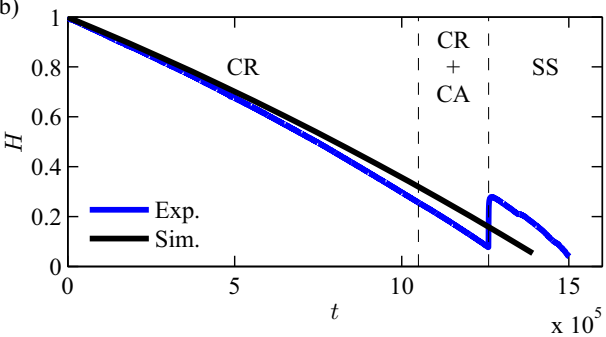

(d)

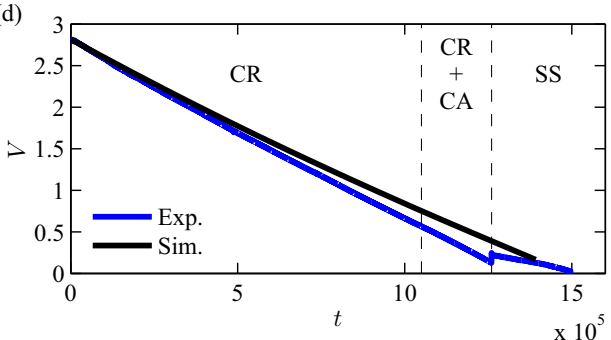

Figure 4: Comparison between experiments and numerical simulations for a water drop evaporating into $\mathrm{N}_{2}$ placed on a heated substrate at $\hat{T}_{w}=40^{\circ} \mathrm{C}$ with $\Delta \hat{T}=14.6{ }^{\circ} \mathrm{C}$. (a) Contact angle $\theta$, (b) drop height $H$, (c) contact radius $R$, and (d) volume $V$. The dashed lines separate the evaporation modes observed experimentally, see subsection 3.2.

tests. The selected time-step size is $\Delta t=5 \times 10^{-3}$. The discussion continues in the dimensionless framework in the following sections.

\subsection{Comparisons with experiments}

The validation of the numerical solutions is more challenging than in the non-heated configuration. This is not only because of the notable numerical difficulty associated with solving seven fully-coupled non-linear PDEs, but also due to the large thermal gradients arising at the interface. Given that $p_{s}$ grows exponentially with $T$, deviations as small as $\pm 2{ }^{\circ} \mathrm{C}$ at high temperatures lead to substantial changes in $m(t)$.

Initially, the flow undergoes a transient process wherein the drop heats up and the vapour disperses in the gas. Simultaneously, the Marangoni effect drives fluid from the contact-line region (hot) towards the apex (cold) and, therefore, induces convection currents in both phases. This transient phase is examined more in detail in subsection 4.2. After this stage, the flow reaches a quasi-steady state wherein the two-phase flow characteristics evolve more gradually with $t$ in accordance to the evaporation timescale. The instantaneous evolution of $\theta, H, R$ and $V$ obtained with the simulations is compared with the experimental data in figure 4. Excellent agreement between experiments and simulations is observed in the initial $\mathrm{CR}$ stage. The average numerical evaporation rate is $m(t)=1.963 \times 10^{-6}, 2.7 \%$ lower than that experimentally observed, i.e. $2.017 \times 10^{-6}$. The deviation increases slightly for $t>10.5 \times 10^{5}$, when the experimental drop evaporates according to the combined $\mathrm{CA}+\mathrm{CR}$ and $\mathrm{SS}$ modes. This is to be expected as in the simulations the drop remains perfectly pinned and therefore the evaporation modes are not comparable at this stage. A more detailed discussion examining the instantaneous $m(t)$ is presented in section 6 .

The second key point in the validation is the comparison between the experimental and numerical interface temperature, shown in figure 5a. Overall, there is again good qualitative and quantitative agreement. The only discrepancies are found at the contact 

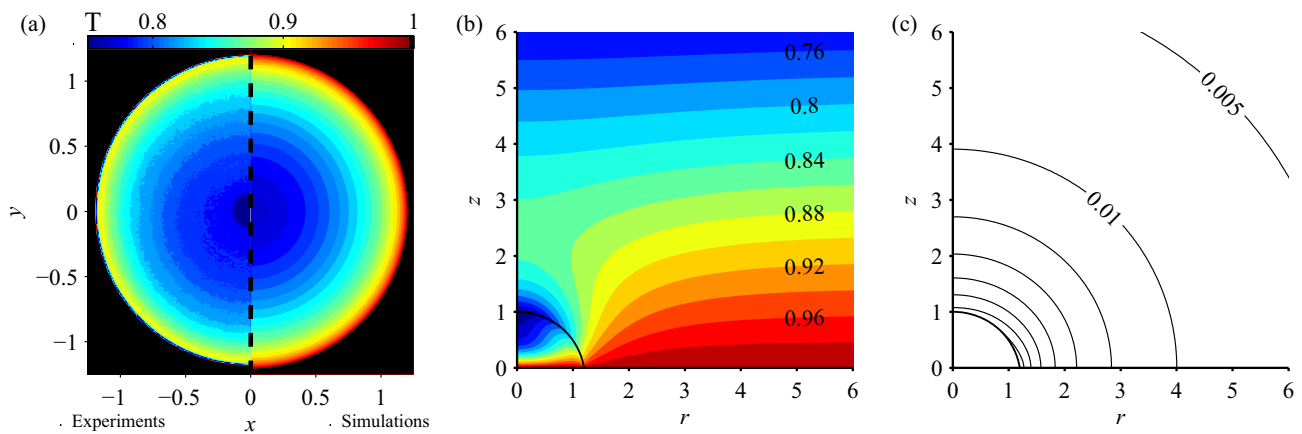

Figure 5: Water drop evaporating into $\mathrm{N}_{2}$ with $\hat{T}_{w}=40^{\circ} \mathrm{C}$. (a) Comparison between the experimental and numerical interface temperature with $H=0.6$, (b) liquid-gas temperature, and (c) vapour mass fraction in the initial stages of the evaporation process.

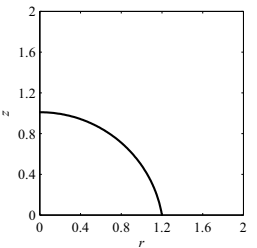

(a)

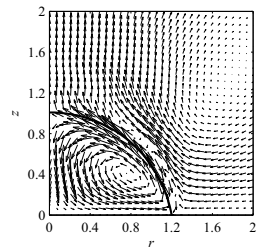

(b)

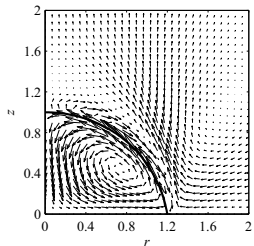

(c)

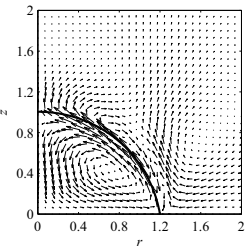

(d)

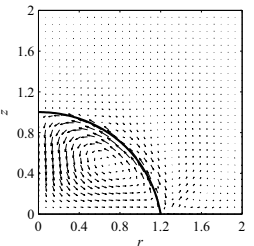

(e)

Figure 6: Initial evolution of the two-phase velocity for a water drop placed on a heated substrate at $\hat{T}_{w}=40^{\circ} \mathrm{C}$ with $\Delta \hat{T}=14.6{ }^{\circ} \mathrm{C}$. (a) Initial conditions, $t=0$, (b) $t=750$ $(0.42 \mathrm{~s}),(\mathrm{c}) t=1.5 \times 10^{3}(0.83 \mathrm{~s}),(\mathrm{d}) t=2.25 \times 10^{3}(1.25 \mathrm{~s})$, and (e) quasi-steady state, $t=3 \times 10^{4}(16.7 \mathrm{~s})$.

line but these result from the fact that the IR emissions are distorted due to the transition from water (high emissivity) to polished copper (very low). As the contact line is approached, the accuracy of the $T$ readings diminishes due to the transition to a different emissivity; otherwise $T \simeq 1$ should be observed at the contact line with the IR camera. Figure $5 \mathrm{~b}$ shows the distribution of $T$ in the bulk of the liquid and gas. The flow and liquid temperature is qualitatively similar to that previously presented by other authors (Mollaret et al. 2004; Hu \& Larson 2005a; Girard et al. 2006; Yang et al. 2014). The gas temperature, not available in the literature, reveals a colder area above the drop due to the evaporative cooling. This thermal dip is progressively corrected as we move away from the drop until, at certain distance $(r>6), T$ becomes $r$-independent and decreases uniformly for increasing $z$. The vapour distribution is presented in figure $5 \mathrm{c}$.

\subsection{Initial drop heating}

To conclude this section on axisymmetric drops, we provide insights into such fundamental question as how a water drop heats up when placed on a hot substrate. This initial stage has received very little attention in the literature. Note that $\mathrm{Hu} \&$ Larson (2002, 2005a,b, 2006) or Girard et al. (2006, 2008a,b); Girard \& Antoni (2008) described the transient drop evolution as a sum of steady-state solutions and, therefore, could not capture the dynamics of the initial stage.

The evolution in time of the two-phase flow velocity is presented in figure 6. During the initial transient phase, the liquid and gas velocities are comparable and significantly 


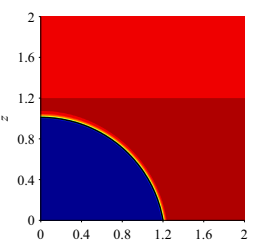

(a)

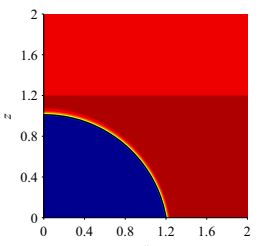

(f)

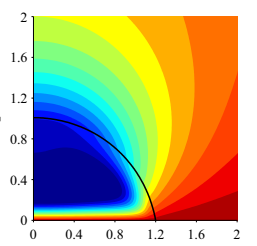

(b)

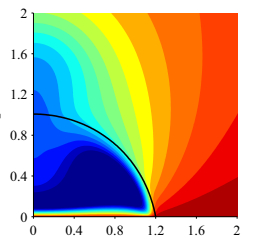

(g)

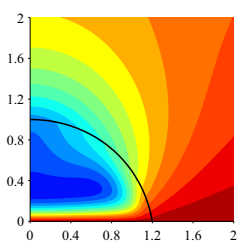

(c)

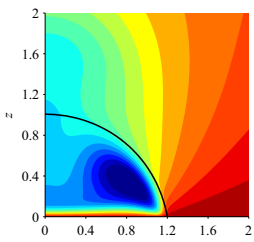

(h)

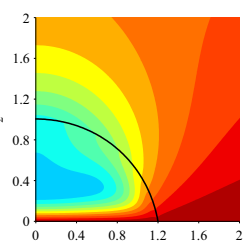

(d)

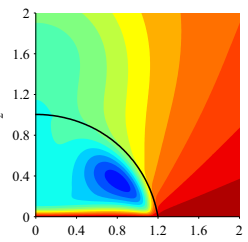

(i)

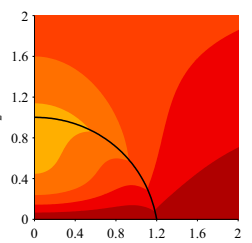

(e)

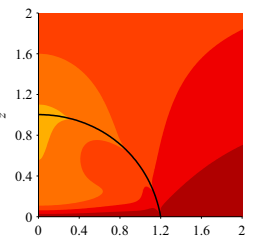

(j)

Figure 7: Transient heating of a water drop at $\hat{T}_{a}$ placed on a heated substrate at $\hat{T}_{w}$. Top row: $\hat{T}_{w}=40^{\circ} \mathrm{C}$ with $\Delta \hat{T}=14.6{ }^{\circ} \mathrm{C}$. (a) Initial conditions, $t=0$, (b) $t=750(0.42$ $\mathrm{s}),(\mathrm{c}) t=1.5 \times 10^{3}(0.83 \mathrm{~s}),(\mathrm{d}) t=2.25 \times 10^{3}(1.25 \mathrm{~s})$, (e) quasi-steady state, $t=3 \times 10^{4}$ (16.7 s). Bottom row: $\hat{T}_{w}=70^{\circ} \mathrm{C}$ with $\Delta \hat{T}=39.8^{\circ} \mathrm{C}$. (f) Initial conditions, $t=0$, (g) $t=750(0.15 \mathrm{~s}),(\mathrm{h}) t=1.5 \times 10^{3}(0.30 \mathrm{~s}),(\mathrm{i}) t=2.25 \times 10^{3}(0.46 \mathrm{~s}),(\mathrm{j})$ quasi-steady state, $t=3 \times 10^{4}(6.08 \mathrm{~s})$.

larger than those of the quasi-steady equilibrium; this is due to the severe thermal gradients resulting from considering an initially cold drop. As the flow converges to the equilibrium state, the gas velocity decreases more rapidly than that of the liquid and ultimately becomes significantly lower (figure 6e). The coupled evolution of the thermal field is depicted in figure 7 for $\hat{T}_{w}=40^{\circ} \mathrm{C}$ and $70^{\circ} \mathrm{C}$ to illustrate how the increasing role of the Marangoni effect influences the dynamics of the process. The strength of thermocapillarity is measured by the Marangoni number, $M a=\hat{\gamma} \hat{R}_{0} \Delta \hat{T}^{*} /\left(\hat{\mu}_{l} \hat{\alpha}_{l}\right)$, where $\hat{\alpha}_{l}$ is the liquid thermal diffusivity and $\Delta \hat{T}^{*}$ is the temperature difference between the contact line and the apex. Calculation of this group shows that $M a$ is 2.5 times larger for $\hat{T}_{w}=70^{\circ} \mathrm{C}$, i.e. the strength of thermocapillarity increases for increasing $\hat{T}_{w}$. Figures $7 \mathrm{a}$ and $7 \mathrm{f}$ show the initial conditions (cold drop) while figures $7 \mathrm{e}$ and $7 \mathrm{j}$ illustrate the quasi-steady $T$ field found after the initial abrupt heating. Between these states, the drop warms up very quickly due to the heat transported from the substrate, across the solid-liquid interface, and surrounding gas, across the liquid-gas interface (see figure $7 \mathrm{~b}$ and $7 \mathrm{~g}$ ). Some of the latter is absorbed by evaporation, which is strongest at the beginning given that $\omega_{t=0}=0$, while the rest begins to increase the interface temperature. The temperature, $T$, drops rapidly in the surrounding gas (its thermal diffusivity is two orders of magnitude larger than that of the liquid) and a thermal gradient arises along the interface triggering the Marangoni convection. Fluid from the contact-line region (at higher $T$ ) is driven along the interface to the apex, which is cooler due to evaporation, and then recirculated vertically downward towards the centre of the drop. At high $\hat{T}_{w}$, the amount of heat associated with this Marangoni convection is significant and leads to the drop being heated through its core, as shown in figure 7g-i. Figure 7h,i reveal that, rather than its center, the coldest part of the drop is a toroidal-like region whose cross-section is approximately centred at $(r, z)=(0.8,0.3)$. At high $\hat{T}_{w}$, therefore, the 
drop reaches thermal equilibrium by means of interior heating due to the prominent role of advection. At lower $\hat{T}_{w}$ on the other hand (see figure $7 \mathrm{~b}-\mathrm{d}$ ) the role played by the Marangoni effect is less significant and, consequently, the drop increases its temperature through its periphery via conduction. In this case, the coldest region coincides with the drop's core. Independently of $\hat{T}_{w}$, the initial thermal adjustment takes place in $<3 \%$ of the drop lifetime, after which the drop enters a quasi-steady phase wherein the flow characteristics evolve very slowly.

\section{Non-spherical drops}

In this section, we consider the dynamics of evaporating, non-axisymmetric drops. As discussed above, the time scale associated with the transport of vapour away from the interface is $O\left(10^{2}\right)$ larger than those associated with the Marangoni-related phenomena that determine the flow dynamics in the drop and surrounding gas. Hence, the system quickly reaches a quasi-steady state wherein the characteristics of the two-phase flow evolve very slowly in time. We exploit this to reduce the computational cost of the $3 \mathrm{D}$ simulations. Rather than resolving the complete domain $\left(20 R_{0}\right)$, which in $3 \mathrm{D}$ is prohibitively costly, we consider smaller subdomains containing the drop and surrounding gas. To close the problem, we make use of our $2 \mathrm{D}$ computations to prescribe the flow conditions in the vertical and upper boundaries. Using this strategy, we can efficiently simulate the quasi-steady drop flow at any point with no need of computing the previous stages that have led to it in $3 \mathrm{D}$.

In the most typical case, an everyday drop is non-spherical from its inception until dry-out. However, there is a second usual scenario that leads to deformed drops. As shown in the experiments (see figure 2d), initially spherical drops are also most likely to become non-spherical after the first contact-line jump occurring towards the end of their lifetime. These two situations are examined in subsections 5.1 and 5.2, respectively. The discussion is supported by comparisons with the axisymmetric flow for $H=0.98$ and 0.3 presented in section 4 . The former, $H=0.98$, represents the idealistic case wherein the drop generated is perfectly spherical and serves to compare with the more realistic situation wherein the drop is deformed from the beginning. For the sake of simplicity, we examine the flow once this has passed the initial transient phase, which provides rationale for choosing the point with $H=0.98$ instead of 1 . This analysis is presented in subsection 5.1. The latter, $H=0.3$, illustrates the drop just before the first contact-line jump and serves to compare with the flow found immediately after the first depinning event. Subsection 5.2 is devoted to this study. It should be noted that these two spherical cases used as reference have also been simulated in 3D to make sure that the results are the same as with the axisymmetric version of the code. The domain size is $L_{x}=L_{y}=2 L_{z}=3 R_{0}$ for $H=0.98$ and $L_{x}=L_{y}=4 L_{z}=3 R_{0}$ for $H=0.3$. The vertical length of the domain is associated with height of the drop investigated in each case. A uniformly-distributed mesh of $250 \times 250 \times 125$ elements is employed in both cases. The time-step size is $\Delta t=5 \times 10^{-4}$. The scalings and governing groups are the same as for the case with $\hat{T}_{w}=40^{\circ} \mathrm{C}\left(\Delta \hat{T}=14.6{ }^{\circ} \mathrm{C}\right)$ presented in section 4 .

\subsection{Elliptical contact area}

In order to generate spherical drops, having a perfectly perpendicular dosing is key. However, in most real configurations the drop deposition is not exactly perpendicular to the substrate. A broad range of factors can be the cause of this, e.g. vertical dosing onto inclined substrates or drops released at certain angle due to setup configurations or even convection currents in the gas, etc. In such cases, and assuming that the drop deposition 
(a)

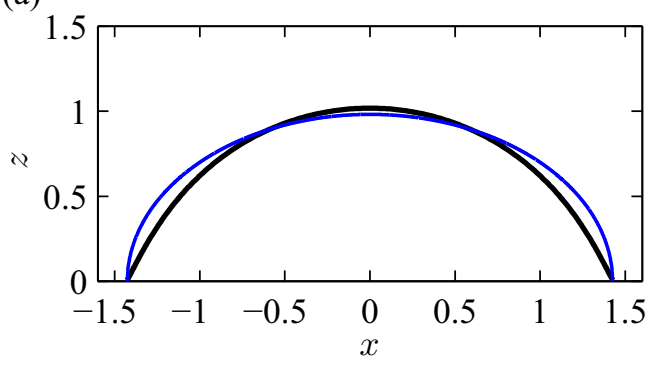

(b)

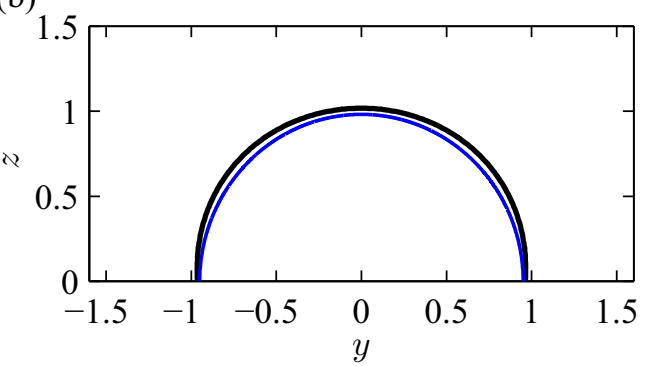

Figure 8: Initial semi-ellipsoidal interface (blue line) and final drop shape (black) along the principal (a) $x z$ and (b) $y z$ planes for the drop with deformed contact area. This drop is defined by the semi-axis lengths of its elliptical contact area, $a=1.4260$ and $b=0.9505$, and its volume, $V=\pi H\left(3 R^{2}+H^{2}\right) / 6$ with $H=0.98$ and $R=1.2$.

is not violent, the resulting contact area is elliptical rather than circular. We, therefore, define the initial drop shape in Cartesian coordinates as follows,

$$
\frac{x^{2}}{a^{2}}+\frac{y^{2}}{b^{2}}+\frac{z^{2}}{c^{2}}=1,
$$

where $a, b$, and $c$ are the semi-axis lengths along the $x, y$, and $z$ directions, respectively. The volume of a semi-ellipsoid $V_{e}$ is given by,

$$
V_{e}=\frac{2}{3} \pi a b c,
$$

while the base circumference $C_{e}$ can be estimated according to Ramanujan's second approximation, i.e.,

$$
C_{e} \approx \pi(a+b)\left(1+\frac{3 h}{10+\sqrt{4-3 h}}\right),
$$

where $h=(a-b)^{2} /(a+b)^{2}$. It is known that the overall evaporation rate of a sessile drop is proportional to its volume (or contact angle) and to the length of its contact line, e.g. Birdi et al. (1989); Rowan et al. (1995); Hu \& Larson (2002). Thus, $a, b$ and $c$ are selected so that the resulting ellipsoidal geometry provides the same volume and base perimeter as those for the spherical drop used as reference, i.e., $V_{e}=\pi H\left(3 R^{2}+H^{2}\right) / 6$ and $C_{e}=2 \pi R$, where $H=0.98$ and $R=1.2$. Equations 5.2 and 5.3 form a set of two relations with three unknowns. The missing expression necessary to complete an independent system is provided by the degree of deformation in the horizontal direction, $\Lambda=a / b$, which is chosen to be $\Lambda=3 / 2$. It follows that the resulting ellipsoidal geometry is defined by $a=1.4260, b=0.9505$, and $c=0.9815$.

Our simulations show that an ellipsoid is not a possible shape for a sessile drop with an elliptical pinned contact line. The initial drop geometry (arbitrarily selected) undergoes a rapid change as surface tension enforces interface surface-area minimization for the given volume. Figure 8 illustrates the initial shape (blue line) together with the final quasisteady drop profile (black). The pinned contact line impedes the formation of a sphere but surface tension drives the interface shape to reach the closest possible configuration. In an attempt to even out the different axis lengths, there is interface pulling along the longest axis, $x$ (figure 8a), while the interface expands in the other two directions, $y$ and $z$ (figure $8 \mathrm{~b}$ ). The interface surface area of the resulting drop is smaller than the area of the ellipsoid but larger than the area of the spherical cap by $1.2 \%$ with the same volume 

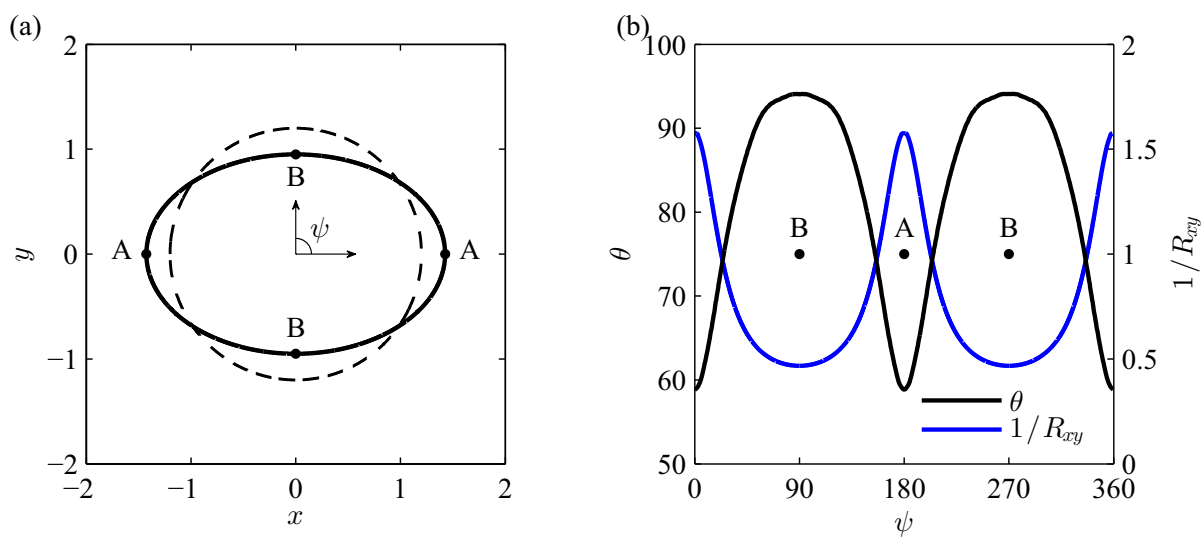

Figure 9: (a) Elliptical contact area of the deformed drop (solid line) along with that of the spherical drop (dashed line) employed for comparison. (b) Contact-angle $\theta$ and horizontal-curvature $1 / R_{x y}$ distribution along the elliptical contact line. This is given in terms of the azimuthal angle $\psi$ defined as shown in (a). Here, $\theta_{\max }=94^{\circ}$ and $\theta_{\min }=59^{\circ}$.

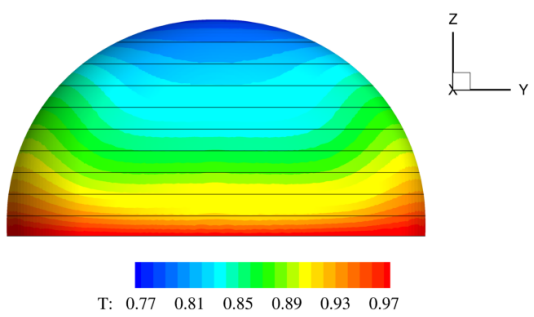

(a)

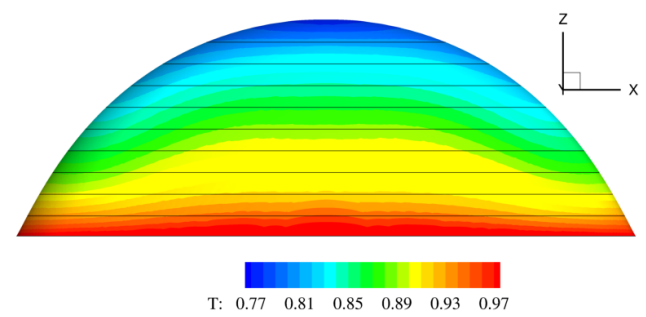

(b)

Figure 10: Interface temperature for the drop with elliptical contact area. The solid black lines are contours of constant $z$ cordinate. (a) Front, and (d) side views.

and base perimeter. It should be noted that this initial shape adjustment is not caused by evaporation. The time interval in which this adjustment takes place is sufficiently short that the volume difference between the initial and the spherical drop shape is negligible $(\Delta V \approx 0.1 \%)$.

Figure 9a depicts how the resulting elliptical contact line of the deformed drop compares with the circular contact line of the reference drop, while figure $9 \mathrm{~b}$ shows the contact-angle distribution along the triple line, which is not constant as in the spherical case. The position along the contact line is given in terms of the azimuthal angle, $\psi$, defined as shown in figure $9 \mathrm{a}$. The contact angle varies between $\theta_{\min }=59^{\circ}$ for the longest horizontal axis $\left(\psi=0^{\circ}\right.$ and $\left.180^{\circ}\right)$ and $\theta_{\max }=94^{\circ}$ for the other $\left(\psi=90^{\circ}\right.$ and $\left.270^{\circ}\right)$. The average contact angle $\bar{\theta}=\int_{\mathrm{CL}} \theta \mathrm{d} l / \int_{\mathrm{CL}} \mathrm{d} l$ is $82^{\circ}$. For a given $V$, the range of contact angle variation throughout the base line, $\Delta \theta=\theta_{\max }-\theta_{\min }$, increases for increasing deformation $\Lambda$. Note that $\Delta \theta=35^{\circ}$ with $\Lambda=3 / 2$ while $\Delta \theta=0^{\circ}$ for $\Lambda=1$ (spherical cap). In addition, for a given $\Lambda, \Delta \theta$ decreases in time due to evaporation. For instance, when the drop has half of the original volume, i.e. $V=V_{e} / 2$, we find that $\Delta \theta=62^{\circ}-31^{\circ}=31^{\circ}$ and $\bar{\theta}=50^{\circ}$. Note that in the limit $V \rightarrow 0$ then $\Delta \theta \rightarrow 0$. In other words, the conclusions are: (i) the more deformed the drop is the larger the range of $\theta$, and (ii) evaporation makes the contact-angle distribution more homogeneous with time. 
It is important to note that the contact-angle distribution is inversely related to the horizontal contact-line curvature $1 / R_{x y}=\left|\left(\nabla_{\mathrm{H}} \cdot \mathbf{n}\right)\right|_{z=0}$, where $\nabla_{\mathrm{H}}=\nabla-\mathbf{n}_{z}\left(\mathbf{n}_{z} \cdot \nabla\right)$ is the horizontal gradient operator, and $\mathbf{n}$ and $\mathbf{n}_{z}$ are the unit vectors normal to the interface and to the lower boundary, respectively. Figure $9 \mathrm{~b}$ illustrates the evolution of $1 / R_{x y}$ between its maximum found at the ends of longest axis $\left(\psi=0^{\circ}\right.$ and $\left.180^{\circ}\right)$, where $1 / R_{x y}=a / b^{2}=1.58$, and minimum at the shortest $\left(\psi=90^{\circ}\right.$ and $\left.270^{\circ}\right)$, where $1 / R_{x y}=b / a^{2}=0.47$. The higher $1 / R_{x y}$ at a certain point, the more this point behaves like a "corner" and, as a result, experiences a greater degree of pulling, resulting in lower $\theta$. To understand the physical mechanism behind this phenomenon, we need to examine Young-Laplace equation, $\Delta p=\sigma\left(1 / R_{1}+1 / R_{2}\right)$. Since gravity has been neglected, the pressure in the liquid and gas are different but essentially constant and, therefore, $\Delta p$ should be equal across any point along the interface. This imposes the constraint that, if the contact-line curvature is large at one point $\left(1 / R_{1}\right)$, the second principal curvature $\left(1 / R_{2}\right)$, which dictates $\theta$ in this case, has to be smaller (larger $\left.\theta\right)$ in order to maintain a constant $\Delta p$. Note that, thanks to the drop symmetry, the principal radii of curvature of the interface at the extreme points $\mathrm{A}$ and $\mathrm{B}$ coincide with radii of curvature of the contact line and the interface contained in the principal vertical plane across the point (directly connected to $\theta$ ). This singularity may not occur for the intermediate points, consideration which is revisited in the more complex case presented in subsection 5.2.

As was mentioned above, the evaporation rate $m(t)$ in spherical drops is known to be dependent on the length of the base circumference $C_{e}$ and volume $V$, which is similar to say that $m(t)$ depends on $C_{e}$ and $\theta$. It is evident that the spherical condition together with $C_{e}$ and $V$ lead to a unique interface surface area and, therefore, to a characteristic $m(t)$. Not surprisingly, this consideration does not hold for deformed drops given that the interface area is not uniquely defined with $C_{e}$ and $V$ only. It is found that $m(t)$ is $2.3 \%$ larger than that for the reference spherical drop even though $C_{e}$ and $V$ are the same in both cases, which is in accordance with the order of surface area increment reported above. This finding is also consistent with the experimental observations by Jansen et al. (2014), who recently reported higher evaporation rates for more elongated droplets.

Figure 10 depicts $T$ along the interface. The temperature ranges from $T=1$ at the triple line to $T=0.77$ at the apex. Given the elliptical contact area, one might have expected $T$ to follow rather closely a distribution of concentric ellipses of decreasing size for increasing $z$. This is not what is observed in the simulations, however. The irregular drop shape leads to the thermal field being deformed in the $z$-direction, as shown in figure 10b. Following the interface shape, the isotherms are stretched downwards along the longest axis. Hence, their vertical location varies depending on the azimuthal position. The curvature and path length towards the apex are also different at each point. This combination of factors gives rise to azimuthal thermal gradients along the interface.

Figure 11 shows the 3D flow within the drop by means of streamlines colored by the local $T$. The flow repeats itself periodically at each quadrant even though the data are from a $3 \mathrm{D}$ simulation of the complete drop. Flow is driven from the contact line to the apex along the interface and then recirculated through the drop core back towards the contact line. However, the streamlines along the interface are bent towards the ends of longest axis, as shown in figure $11 \mathrm{~b}$, due to the azimuthal dependence of $\nabla_{\mathrm{s}} T$; the dashed arrows show the direction of the emerging azimuthal velocity components. This effect also induces azimuthal currents in the interior of the drop, which have been isolated for the first quadrant in figure 11 for clarity of presentation. At each quarter, the current's origin coincides with the center of the vortex at the vertical plane across the shortest axis of the ellipse, i.e. $y z$ plane (figure 11d), and flows into the center of the second fundamental vortex generated along the longest axis, i.e. $x z$ plane (figure 11c). Each 


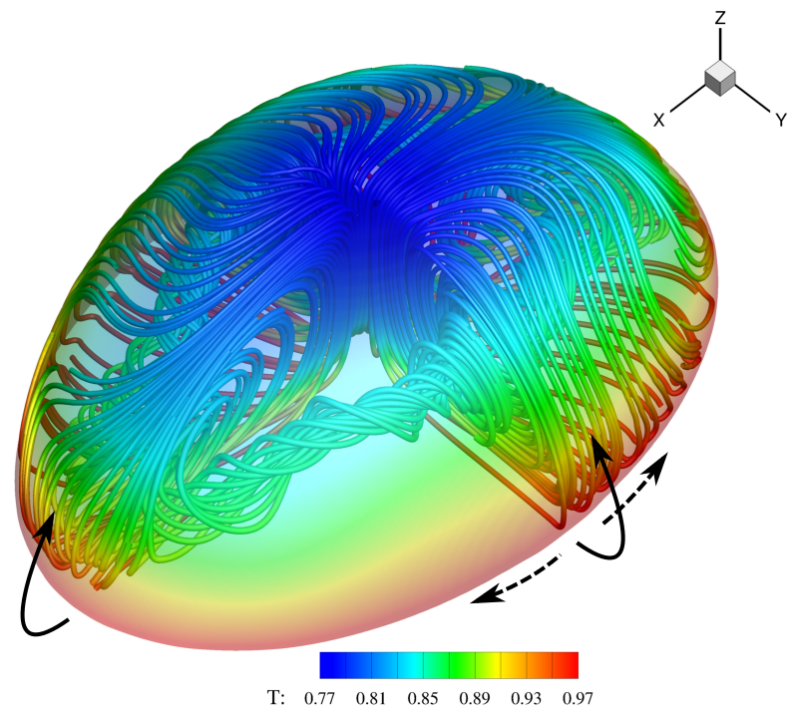

(a)

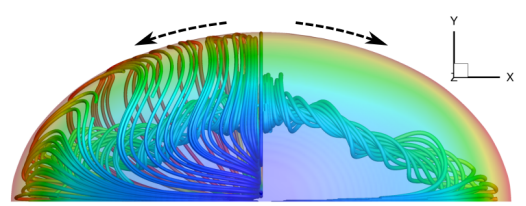

(b)

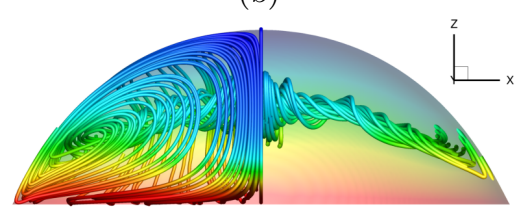

(c)

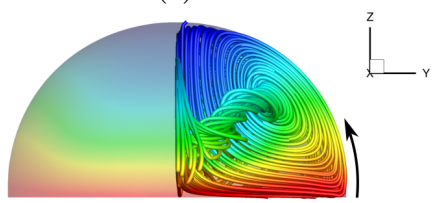

(d)

Figure 11: Three-dimensional flow within the drop with elliptical contact area defined in figure 9. The interface and streamlines are colored by temperature. (a) 3D, (b) top, (c) front, and (d) side views. The solid and dashed arrows indicate the direction of the primary interface flow and secondary azimuthal currents, respectively.

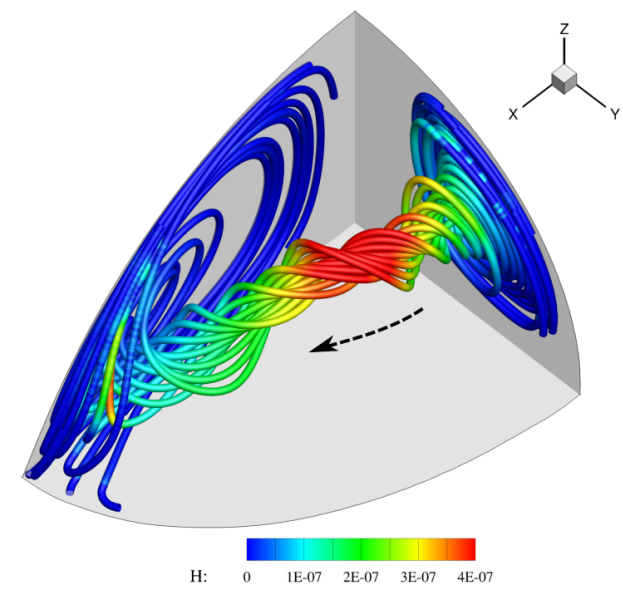

Figure 12: Local volumetric helicity $\mathscr{H}=\mathbf{u} \cdot(\nabla \times \mathbf{u})$ for the azimuthal current emerging in the deformed drop with elliptical contact area. The arrow shows the direction of motion.

stream, therefore, is a bent toroidal-like feature, transporting liquid from areas located in perpendicular planes and at different heights.

In order to characterize the torroidal currents more quantitatively, the local volumetric helicity $\mathscr{H}=\mathbf{u} \cdot(\nabla \times \mathbf{u})$ is presented in figure 12 . Note that $\mathscr{H}$ is large when the flow velocity $\mathbf{u}$ and the vorticity $(\nabla \times \mathbf{u})$ are not only large but also parallel, which makes $\mathscr{H}$ a very useful variable to provide a measure of the current's azimuthal strength. At 
(a)

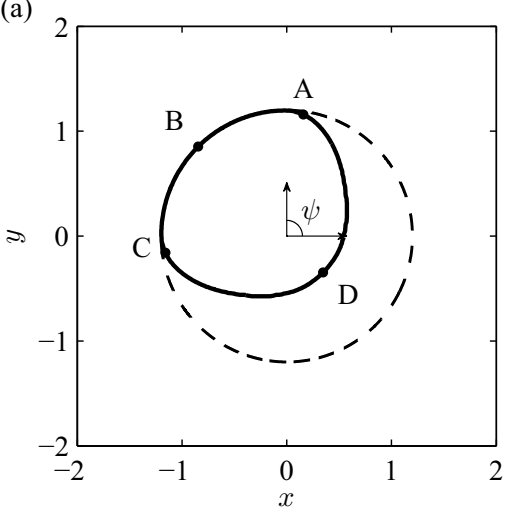

(b)

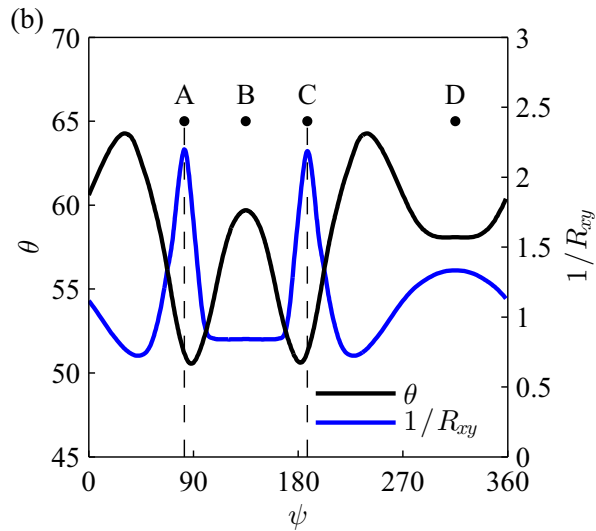

Figure 13: (a) Irregular contact area of the deformed drop found after the first contact jump (solid line) compared with that of its precursor spherical drop (dashed line) with $H=0.3$ and $R=1.2$. (b) Contact-angle $\theta$ and horizontal-curvature $1 / R_{x y}$ distribution along the contact line. This is given in terms of the azimuthal angle $\psi$ defined as shown in (a). Here, $\theta_{\max }=64^{\circ}$ and $\theta_{\min }=51^{\circ}$. The key points are $\mathrm{A}\left(\psi_{A}=82^{\circ}\right), \mathrm{B}\left(\psi_{B}=135^{\circ}\right)$, $\mathrm{C}\left(\psi_{C}=188^{\circ}\right)$ and $\mathrm{D}\left(\psi_{D}=315^{\circ}\right)$.

the vertical planes $\mathscr{H}=0$ because the $\mathbf{u}$ components perpendicular to the planes are 0 . Note that for each plane the perpendicular velocity on one side is in the opposite direction to the perpendicular velocity from the other side. For instance, at the $y z$ plane, $\left(\mathbf{u} \cdot \mathbf{n}_{x}\right)_{x \rightarrow 0}^{x<0}=-\left(\mathbf{u} \cdot \mathbf{n}_{x}\right)_{x \rightarrow 0}^{x>0}$, and, therefore, $\left(\mathbf{u} \cdot \mathbf{n}_{x}\right)_{x=0}=0$. Similarly for the $x z$ plane, $\left(\mathbf{u} \cdot \mathbf{n}_{y}\right)_{y=0}=0$ given that $\left(\mathbf{u} \cdot \mathbf{n}_{y}\right)_{y \rightarrow 0}^{y<0}=-\left(\mathbf{u} \cdot \mathbf{n}_{y}\right)_{y \rightarrow 0}^{y>0}$. As the fluid departs from the $y z$ plane, there is an increment in the flow's $\mathscr{H}$ until it reaches a maximum and then decays to become 0 again at the $x z$ plane. Thus, a particle following this path will probably accelerate during the first stage and slow down at the end. The speed of these currents is approximately one order of magnitude lower than the maximum flow speed, which is found at the interface, and, therefore, comparable to the speed of the flow in the drop's core.

\subsection{Irregular contact area}

We now study the case wherein an evaporating spherical drop becomes deformed after the first contact-line jump, which is typically observed in the experiments at $t=0.7 t_{t o t}$. In the range of temperatures investigated in the laboratory, the contact-line motion begins when a certain critical disequilibrium point is reached in the dynamics. The critical conditions change slightly with $\hat{T}_{w}$ but, in the range of temperatures examined in the laboratory $\left(40^{\circ} \leqslant \hat{T}_{w} \leqslant 70^{\circ}\right)$, these fall in the range $22^{\circ} \leqslant \theta_{c} \leqslant 26^{\circ}$, which in terms of drop height becomes $0.37 \mathrm{~mm} \leqslant \hat{H}_{c} \leqslant 0.44 \mathrm{~mm}$ (figure 3 ), and $0.24 \leqslant H_{c} \leqslant 0.28$ in dimensional, and dimensionless form, respectively. For sake of simplicity, we round $H_{c}$ and consider that the drop depinning occurs when $H=0.3$.

The experiments show that during the 3D contact-area motion, a section of the triple line remains anchored to its original position (top-left quadrant in figure $2 \mathrm{~d}$ ) while the rest suddenly moves to regain equilibrium by reducing the contact area. In our simulations, we generate the deformed drop in a similar way (see figure 13a). Starting from an axisymmetric flow with $H=0.3$ and $R=1.2$ in 3D (dashed line in figure 13a), the contact line is allowed to move freely everywhere except in the second quadrant, $90^{\circ} \leqslant \psi \leqslant 180^{\circ}$, where it is pinned. Consequently, surface tension brings the MCL towards its new pinned 
position (solid line in figure 13a) to satisfy interface area minimization. The new pinned position has been selected approximating experimental observations, and the model implemented to allow contact-line motion, which is not presented here to maintain the focus of the investigation, is described in section 6 . Note that the focus here is on elucidating the dynamics of evaporation from a deformed drop; thus modelling the complex motion of the contact line that brings about this deformation is beyond the scope of this work.

Contrary to the previous drop geometry with elliptical contact area, this more irregular drop only presents one vertical plane of symmetry, namely that across points B and D in figure 13a. The resulting $\theta$ distribution, shown in figure 13b, is more intricate but follows similar principles. During the readjustment, $\theta$ increases from $28^{\circ}$ (spherical drop) to $51^{\circ} \leqslant \theta \leqslant 64^{\circ}$, which is in excellent agreement with the range recorded experimentally, as shown in figure 3a. Once again, $\theta$ is related to the contact-line curvature $1 / R_{x y}$ as the points of minimum $\theta$ coincide fairly well with the locations where $1 / R_{x y}$ is maximum, i.e. points $\mathrm{A}$ and $\mathrm{C}$, and vice versa. Note that $\theta$ and $1 / R_{x y}$ are perfectly in phase at $\mathrm{B}$ and $\mathrm{D}$ whereas there is a small mismatch for $\mathrm{A}$ and $\mathrm{C}$. The reason lies in the drop symmetry. The angles $\theta_{B}$ and $\theta_{D}$ result from the equilibrium of interfacial forces acting on each side of the line $\mathrm{BD}$, which are symmetrical. In the case of $\mathrm{A}$ and $\mathrm{C}$, however, the interface forces on each side of the line that connects these points are different due to the drop shape. Note that $R_{x y}$ corresponds to one of the principal interface radii of curvature for B and D but not necessarily for A and C.

The depinning of the drop occurs when it is sufficiently out of equilibrium, that is, when its interface surface area is very large in comparison with its volume. We find that for the same $V$, the geometrical readjustment leads to an interface whose area is significantly smaller $(30.4 \%)$ than the interface area of the spherical drop found right before the depinning event. In a similar manner, the evaporation rate $m(t)$ decreases by the same order of magnitude: $m(t)$ is $20.3 \%$ lower after the contact-area jump. The reasons why there is a relative mismatch between the change of surface area and evaporation rate appear to be associated to the rapid transient nature of the flow. Careful analysis of the instantaneous $m(t)$ reveals that, during the transient stage, $m(t)$ in the irregular drop decreases at a rate $O(1)$ faster than that in the spherical drop used as reference. This indicates that the deviation between the change in area and evaporation rate (10.1\%) is being corrected with time and, therefore, probably a short-term consequence of the complex transient flow. It should be noted that a complete correction is unlikely given that the contact-line motion also entails a significant redistribution of drop's thermal field, along with a change in the strength of the Marangoni effect. Hence, a residual mismatch is expected but, once again, the surface area appears to be the leading factor in dictating changes in $m(t)$.

During the contact-line jump, the interface motion severely deforms the preceding axisymmetrical flow. This loses its quasi-steady condition, triggering a rapid transient stage until another equilibrium state is achieved for the new geometrical configuration. The 3D streamlines and $T$ distribution within the drop during this transitional phase are illustrated in figure 14. The Marangoni force develops a preferential direction, line $\mathrm{BD}$ in figure 13a, along which warm liquid is transported from $\mathrm{D}$ towards $\mathrm{B}$, and then recirculated downward and back along the periphery via the points $\mathrm{A}$ and $\mathrm{C}$. Consequently, a pair of counter-rotating vortices emerge in the bulk flow, see figure 14. These are centered in the colder regions and are essentially parallel to the horizontal plane. The strength of these vortices diminishes with time as the interface temperature is homogenized by the central stream of warm fluid as well as by the the heat being transported towards the cold vortices from the contact line due to both conduction and convection. Eventually the vortices disappear, along with the preferential direction of the Marangoni 


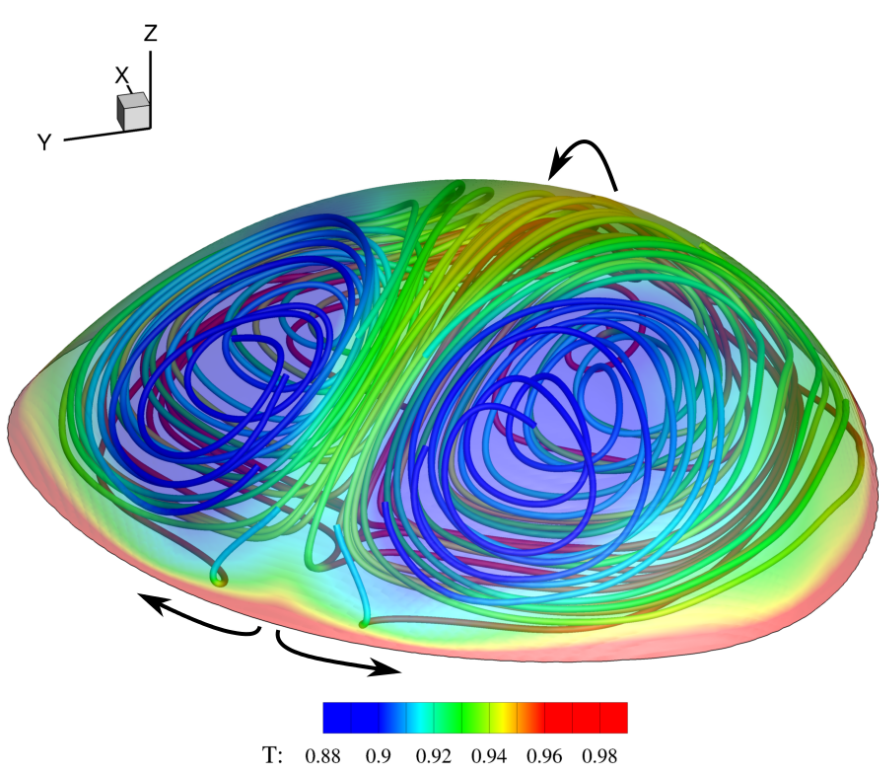

(a)

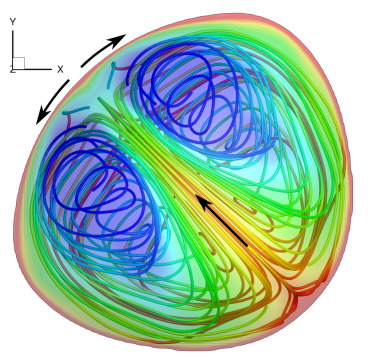

(b)

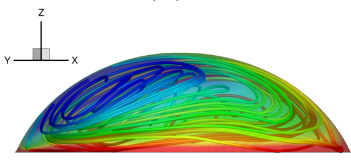

(c)

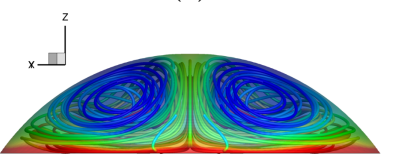

(d)

Figure 14: Three-dimensional flow and temperature distribution in the irregular drop found after the first contact-line jump defined in figure 13. (a) 3D, (b) top, (c) side, and (d) front views. The arrows illustrate the direction of motion.

(a)

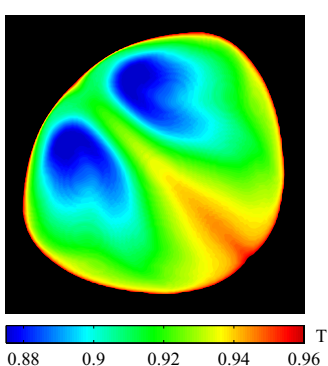

(b)

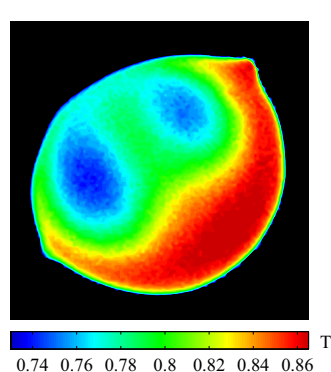

Figure 15: Interface temperature showing the pair of counter-rotating vortices emerging during the rapid thermal adjustment in an irregular drop: (a) Simulations, (b) IR image showing experimentally the emergence of counter rotating vortices in evaporating drops with $\hat{T}_{0}=10{ }^{\circ} \mathrm{C}$ and $\hat{T}_{w}=70{ }^{\circ} \mathrm{C}$.

convection, and the drop reaches its new quasi-steady equilibrium wherein the the apex is the coldest region and the interface flow is mainly radial towards the apex. Azimuthal components also emerge in this new quasi-steady equilibrium but these are weaker than those reported for the drop with an elliptical contact area given that the interface geometry is less deformed. The interface temperature distribution during the intermediate transient stage is depicted in figure 15a. Capturing these vortices in the laboratory is very difficult due to the relatively small range of the $T$ and because the transient stage after depinning for our chosen experiment lasts a very short period of time $(\sim 0.8 \mathrm{~s})$. Note that the drop volume at this point is quite small and, thus, any imbalance is quickly 
corrected by the substrate, whose dominance over the drop thermal field increases for decreasing $V$. However, similar vortices are clearly observed during the initial transient stage of irregularly-shaped colder drops placed on the heated substrate, see figure 15b. By reducing the initial liquid temperature, the vortices emerge more vigorously and persist longer times, which makes it easier to record them in the laboratory. The qualitative agreement between the numerical simulations (figure 15a) and the experiments (figure $15 \mathrm{~b}$ ) is excellent. Given the importance of these vortices in regulating the initial dynamics of evaporating drops, a follow-up investigation is being undertaken, the focus of which is on the detailed numerical and experimental study of these and similar 3D features emerging in non-spherical drops with more diverse and complex contact areas.

\section{Drop evaporation with a moving contact line}

In general, the simulation of moving contact lines (MCL) is rather complicated. The state of the art of the subject has been recently reviewed by Sui et al. (2014). To date, only Murisic \& Kondic (2011), via the lubrication approximation, and Karapetsas et al. (2012) have numerically considered the evaporating sessile drops with a MCL, but as pointed out by Sui et al. (2014), they used a finite-element method for a single fluid with a free surface. Thus, DNS of a fully coupled two-phase flow with a MCL and phase change remains a challenge (Sui et al. 2014). In addition, it should be noted that Karapetsas et al. (2012) examined an intermediate pinned-receding contact line configuration rather than the pure CA mode, which makes their results inadequate for comparison with either of the limiting modes. The versatility of the DI method allows simulating the pure CA mode with minor changes as the interface thickness circumvents the stress singularity at the MCL (Seppecher 1996; Jacqmin 2000). Here, we present DNS of our fully-coupled two-phase model for a drop evaporating in the pure CA mode and compare the results with those of the same drop evaporating according to the CR mode. The latter correspond to those presented during the model validation against the experimental data in section 4. The governing groups, mesh, initial conditions, etc., are kept the same as those listed in section 4. The interface evolution in the CR and CA modes is presented in figures 16a and $16 \mathrm{~b}$, respectively. In what follows, the initial transient stage $\left(<3 \%\right.$ of $\left.t_{t o t}\right)$ is left out for sake of simplicity restricting the discussion to the quasi-steady flow

In other typical MCL problems, such as drop coalescence or drop spreading, predicting the contact-line motion is a challenge intimately related to the flow regime given by the Ohnesorge number $O h=\hat{\mu}_{l} / \sqrt{\hat{\rho}_{l} \hat{\sigma}_{0} \hat{R}_{0}}$. Very low values of $O h$ denote flows wherein inertia and/or surface-tension forces are dominant over viscous stresses. Under such conditions, the use of the currently available MCL models is justified provided the grid spacing in the contact line region is small enough for the viscosity and surface tension to be the dominant forces. In other words, the mesh must be able to resolve the flow not only on the macroscale, but also simultaneously on the microscale, which is easily below $O\left(10^{-4}\right)$ of the macroscale (Sui et al. 2014). The reader should note, however, that this is not necessary to solve the CA evaporation mode under consideration. The generally tremendous complexity of predicting the contact-line motion is greatly simplified here due to the fact that this is very slow and a direct consequence of evaporation only, even though the $O h=O(0.001)$ number suggests an inertia-capillary regime. This is demonstrated as follows. It is trivial to show that the volume of a spherical cap $\left(0^{\circ}<\theta \leqslant 90^{\circ}\right)$ can be calculated in terms of $R$ and $\theta$ as $V=\pi C_{1}\left(3+C_{1}^{2}\right) R^{3} / 6$, where $C_{1}=(1-\cos \theta) / \sin \theta$. Since $C_{1}$ is constant in the CA mode, it follows that $(\mathrm{d} V / \mathrm{d} t) /(\mathrm{d} R / \mathrm{d} t)=C_{2} R^{2}$, with $C_{2}=\pi C_{1}\left(3+C_{1}^{2}\right) / 2$. In other words, the representation of the ratio of evaporation rate 

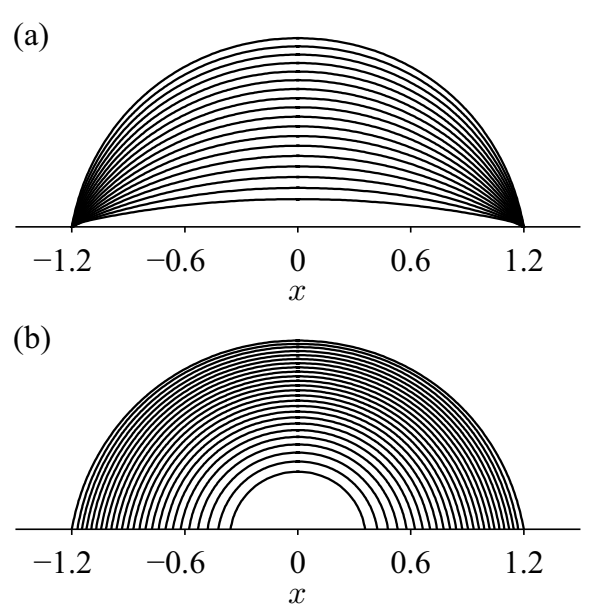

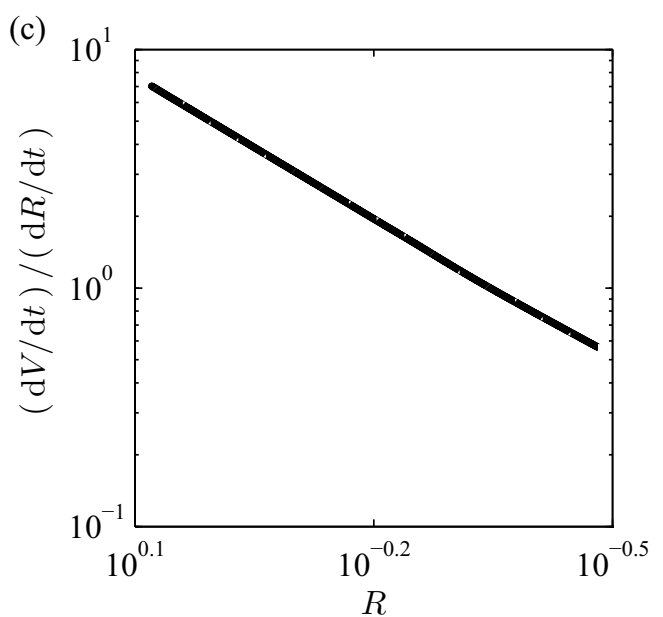

Figure 16: Interface evolution for a drop evaporating according to the (a) CR, and (b) CA pure modes with $\hat{T}_{w}=40{ }^{\circ} \mathrm{C}$ and $\Delta \hat{T}=14.6{ }^{\circ} \mathrm{C}$. The time interval between interface snapshots is $\Delta t=7.5 \times 10^{4}$; (c) Ratio of the evaporation rate to the contact-line speed $(\mathrm{d} V / \mathrm{d} t) /(\mathrm{d} R / \mathrm{d} t)$ versus instantaneous base radius $R$ for the drop evaporating in the CA mode (logarithmic scales).

to contact-line speed, $(\mathrm{d} V / \mathrm{d} t) /(\mathrm{d} R / \mathrm{d} t)$, versus the instantaneous base radius, $R$, should be linear in logarithm scale. Figure 16c shows that this is effectively observed in the CA case under consideration. Also, if evaporation dictates the contact-line movement, when $(\mathrm{d} V / \mathrm{d} t) \rightarrow 0$ then $(\mathrm{d} R / \mathrm{d} t) \rightarrow 0$. This criterion is employed to numerically test that a drop wherein the liquid loss is artificially switched off ( $S=0$ in equation 2.8) results into a motionless contact line. Hence, it is concluded that the inertial-capillary regime suggested by the $O h$ in the context of spreading/coalescence is not applicable for sessile drops evaporating slowly in the CA mode. It is therefore not necessary to go to the microscale in order to predict the contact-line motion.

The instantaneous drop evolution for the $\mathrm{CR}$ and $\mathrm{CA}$ modes is compared in figure 17. Figure 17 a demonstrates the capacity of the DI model to maintain $\theta$ constant (CA mode) while $R$ recedes in a weakly non-linear fashion (figure 17c). The height $H$ decays more progressively in the CA mode than in the CR mode (figure 17b). Similarly to what Picknett \& Bexon (1977) predicted for the isothermal problem, the drops have almost the coincident volume $V$ during a relatively large period of time, $t \sim 6 \times 10^{5}$, point from which the deviation in $V$ becomes more evident. The CA mode leads to lower evaporation rates and, therefore, longer lifetimes, once again in agreement with Picknett \& Bexon (1977) and posterior works.

At first sight, the volume $V$ seems to vary linearly in the CR mode. However, a detailed analysis of the curves shows that $V(t)$ is non-linear for both the CR and CA cases. The variation of the evaporation rate $m(t)$ with time is depicted in figure 18a . In agreement with Picknett \& Bexon (1977), we find that, in the CA mode, the rate is less and declines much more evenly over the whole life of the drop. In the CR mode, however, Picknett \& Bexon (1977) predicted an essentially linear evolution of $m(t)$ in time (for the range of $\theta$ considered here) purely based on geometrical factors. Our computations, which also include non-isothermal dynamics, clearly reveal a non-linear tendency. Comparatively, we find that the initially-linear $m(t)$ decay is mitigated towards the end of the drop 
(a)

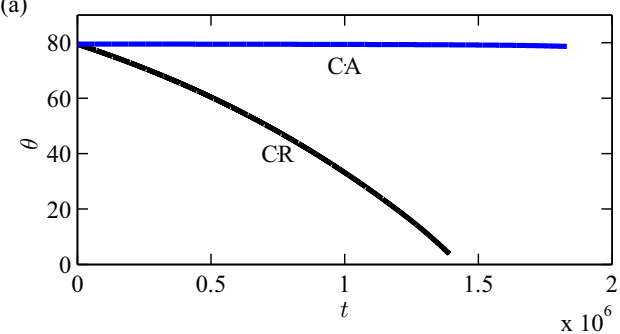

(c)

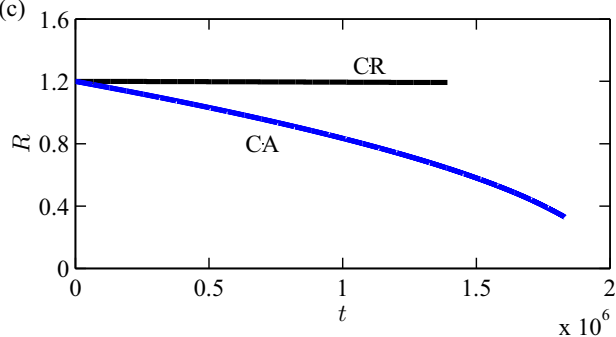

(b)

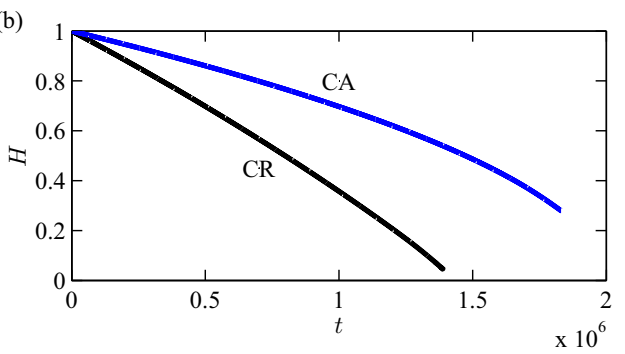

(d)

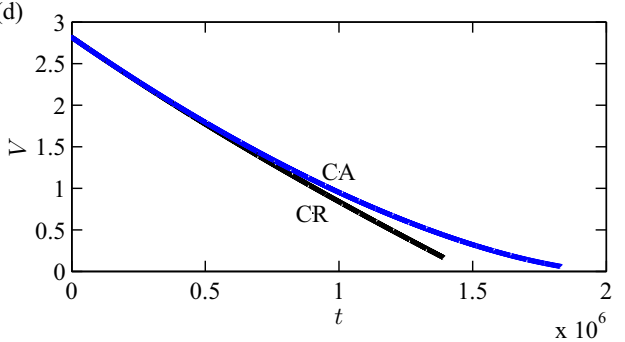

Figure 17: Instantaneous drop parameters for a water drop evaporating according to the $\mathrm{CR}$ and CA pure modes with $\hat{T}_{w}=40{ }^{\circ} \mathrm{C}$ with $\Delta \hat{T}=14.6^{\circ} \mathrm{C}$. (a) Contact angle $\theta$, (b) drop height $H,(\mathrm{c})$ contact radius $R$, and (d) volume $V$.

lifetime, suggesting that there is some temperature-associated phenomenon becoming more relevant at later times which contributes positively to $m(t)$.

Figure $18 \mathrm{~b}$ illustrates the instantaneous area-averaged interface temperature $\bar{T}_{i}=$ $\int T_{i} r_{i} \mathrm{~d} l_{i} / \int r_{i} \mathrm{~d} l_{i}$ for the CR and CA modes, where $T_{i}$ is the interface temperature at the radial coordinate $r_{i}$ over a differential interface arc of length $\mathrm{d} l_{i}$. As presumed, in the CR mode the average interface temperature $\bar{T}_{i}$ increases as the drops becomes thinner and, consequently, the local saturation pressure $p_{s}$ is higher on average. Given that $p_{s}$ is an exponential function of $T$, the rate of decay in $m(t)$ due to the geometrical readjustment is partially counteracted by the evaporation-rate enhancement associated with the transient thermal field. This appears to be a reasonable explanation for the final flattening of $m(t)$, but a more detailed parametric investigation is necessary to provide a conclusive answer to this conjecture.

However, it is very important to realize that, in the CA mode, the average interface temperature essentially remains constant $\left(\bar{T}_{i}=0.819\right)$ as the drop reduces its size (figure 18b). Although this might not be intuitive at first, it is logical if we bear in mind that the MCL in the CA mode leads intermediate drop profiles which maintain the same geometrical ratio. It follows that, in essence, the rate of decay in $m(t)$ in the CA mode is due to geometrical factors only, and not affected by transient changes in the thermal field as in the CR mode. Hence, transient thermal changes during the drop evaporation contribute to increase $m(t)$ in the CR mode (the average drop temperature rises) but are irrelevant in the $\mathrm{CA}$ mode (the average drop temperature remains constant), which allow us to arrive to a conclusion which Picknett \& Bexon (1977) could not provide: the higher the substrate temperature, the larger the difference between the lifetime in the CA (longer) and CR (shorter) evaporation modes. In other words, for increasing temperature, the evaporation rate increases more rapidly in the $\mathrm{CR}$ mode than in the CA mode.

To conclude, we also present in figure $18 \mathrm{~b}$ the instantaneous interface surface area $A_{i}$. In the CR mode, $A_{i}$ decreases non-linearly approaching asymptotically the theoretically 
(a)

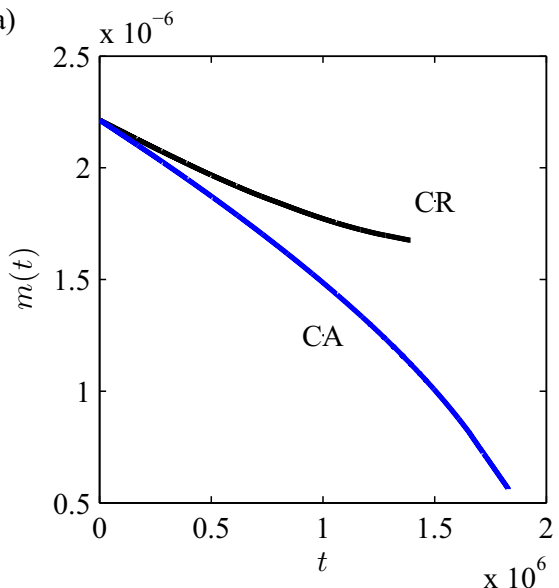

(b)

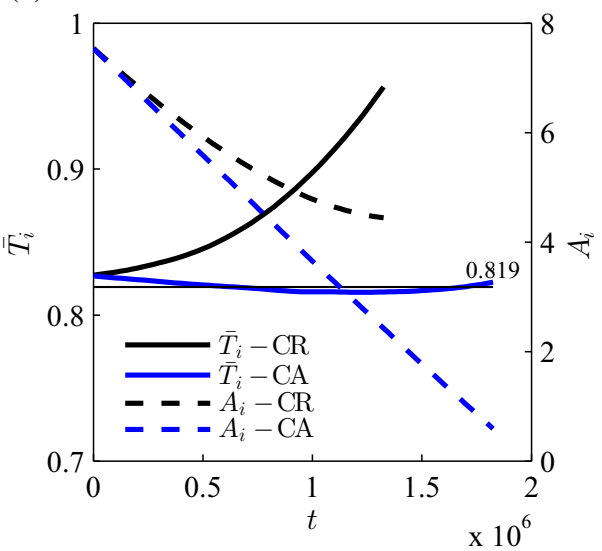

Figure 18: Instantaneous drop evaporation in the CR versus CA modes with $\hat{T}_{w}=40{ }^{\circ} \mathrm{C}$ and $\Delta \hat{T}=14.6{ }^{\circ} \mathrm{C}$. (a) Evaporation rate $m(t)$; (b) Area-averaged interface temperature $\bar{T}_{i}$, and interface surface area $A_{i}$.

final interface area, which is finite, i.e. as $V \rightarrow 0$ then $A_{i} \rightarrow \pi R_{0}^{2}$. In the CA mode, on the other hand, the $A_{i}$ decay is more pronounced, effectively linear, and approaches zero, i.e. as $V \rightarrow 0$ then $A_{i} \rightarrow 0$.

\section{Conclusions}

Despite the fact that most evaporating sessile drops in real-life applications are nonspherical or display a number of 3D phenomena, e.g. the thermocapillary instabilities recently reported by Sefiane et al. (2008), the theoretical and numerical work available in the literature has been restricted to the $2 \mathrm{D}$ axisymmetric problem for sake of simplicity. It should be noted that Karapetsas et al. (2012) examined the instabilities reported by Sefiane et al. (2008), but their study was restricted to linear theory in 3D. In this investigation we present a novel fully-coupled two-phase model based on the diffuse-interface method to conduct 3D direct numerical simulations of deformed drops evaporating on a heated substrate. In addition, both the pinned and moving contact line configurations are addressed. To the best of our knowledge, this is the fist time that such investigation is presented.

The discussion begins in section 4 with the model validation against the data from a set of experiments, presented in section 3, wherein the evaporation of water drops in controlled environments is investigated by means of optical and infra-red techniques. The substrate temperature is varied in the range of $40-70^{\circ} \mathrm{C}$. Good quantitative agreement is achieved for the instantaneous drop parameters (figure 4 ) as well as interface temperature (figure 5). The initial transient heating in the 2D axisymetric problem is compared for different levels of temperature. It is observed that, as the temperature increases, the increasing prominence of the Marangoni convection leads the heating of the drop to occur through its core, mechanism which is referred to as interior heating in section 4.2. At moderate heating, on the other hand, the mechanism of heat transfer is not significantly affected by the Marangoni effect and the drop reaches thermal (quasi) equilibrium via peripheral conduction.

The body of this study is concerned with the analysis of irregular drops in 3D, discus- 
sion which is presented in section 5. Two scenarios are studied. Firstly, we consider the case of a drop that is deformed at its inception due to non-perpendicular dosing onto the substrate, i.e. the drop's contact area is elliptical. The resulting drop geometry is very complex and its interface thermal distribution leads to the emergence of Marangoni stresses perpendicular to the radial direction across the apex. As a result, azimuthal current develop in the drop's bulk flow (figure 11). These drive liquid to the centers of recirculation vortices established on the vertical plane across the longest ellipse's axis from the centers of the vortices appearing on the other principal vertical plane. In a second case, we examine the dynamics of the typical irregular drop resulting from the first contact-line depinning undergone by an initially spherical drop. This phenomenon is typically observed in the experiments towards the end of the drop's lifetime. The abrupt contact-line readjustment experienced by the drop to regain equilibrium induces an intermediate transient stage in the flow. This rapidly evolves to reach a different quasi steady-state equilibrium for the new geometrical configuration at the next pinning location. During this stage, the Marangoni convection adopts a preferential direction which induces a pair of self-excited counter-rotating vortices within the drop (figure 14). These vortices play a prominent role in the bulk velocity field and transient mechanism of heat transfer. The drop displays two lateral cold spots (centered at the votices) until the new thermal equilibrium is attached and the apex becomes the coldest location once again. These previously-unknown phenomena present excellent agreement with the experimental observations where the emergence of the vortices is captured by the infra-red camera (Figure 15). Given the importance of these transient features, a parallel investigation is being undertaken, the focus of which is on the vortices' emergence and dynamics.

For a given level of heating, it is known that the evaporation rate of a spherical drop depends on its volume and base-perimeter length. Unsurprisingly, this conclusion does not hold for deformed drops, as the length of the base perimeter and volume are not enough to define a unique drop interface. The evaporation rate is found to change in the same order of magnitude as the interface-surface area even when the volume and/or circumference are maintained. In addition, deformed drops display intricate wettability distributions, see figures 9 and 13, wherein the local contact-angle in one drop can vary in a broad range (up to $\sim 40^{\circ}$ in the cases studied here). It is shown that these distributions are intimately related to the curvature of the contact line, which provides an easy criterion to quickly estimate the local wettability distribution of complex 3D drops. In general, the curvature is inversely proportional to the contact angle, i.e. points where the contact angle is minimum for the points where the contact-line curvature is maximum and vice versa. Other conclusions are that (i) the more deformed the contact-area is, the wider the range of its contact angle (ii) the contact-angle distribution becomes more homogeneous in time due to evaporation.

To conclude, section 6 is devoted to compare the instantaneous evolution of a pinned drop with that of the same drop evaporating according to the pure constant-angle mode and, therefore, with a moving contact line. This serves to demonstrate the versatility and capacity of our two-phase 3D model to address contact-line dynamics with minor modifications. Examining the isothermal problem, Picknett \& Bexon (1977) concluded that the constant-angle mode leads to a lower evaporation rate and, therefore, a longer lifetime. The same is true in the heated problem. However, it is found that the average interfacial temperature remains constant for the most part of the drop lifetime in the constant-angle mode, while this increases in the constant-radius mode as the drop becomes thinner. Thus, it is concluded that, for increasing temperature, the evaporation rate increases more rapidly in the constant-radius mode than in the constant-angle mode, 
which means that the difference between the lifetime of the former (shorter) and latter (longer) modes increases for increasing level of heat.

Acknowledgments: The authors gratefully acknowledge the financial support of the EC through the ThermaPOWER project (IRSES GA-2011-294905), and EPSRC through the DTG grant (EP/P50550X/1) and Boiling in Microchannels project (EP/K00963X/1). This work made use of the facilities of ARCHER, the latest UK National Supercomputing Service. ARCHER provides a capability resource to allow researchers to run simulations and calculations that require large numbers of processing cores working in a tightlycoupled, parallel fashion. The ARCHER Service is based at Edinburgh and is provided by the EPSRC, NERC, EPCC, Cray Inc. and The University of Edinburgh. Further access to the facilities of ARCHER was provided through the ARCHER Resource Allocation Panel, project number e174. The optimisation of the DIM Solver is also being currently funded under the ARCHER Embedded Computational Science and Engineering (eCSE) Service.

\section{REFERENCES}

AJAev, V. S. 2005 Spreading of thin volatile liquid droplets on uniformly heated surfaces. Journal of Fluid Mechanics 528, 279-296.

Anderson, D. M. \& Davis, S. H. 1995 The spreading of volatile liquid droplets on heated surfaces. Physics of Fluids 7 (2), 248-265.

Anderson, D. M., McFadden, G. B. \& Wheeler, A. A. 1998 Diffuse-interface methods in fluid mechanics. Annual Review of Fluid Mechanics 30, 139-165.

Ascher, U. M., Ruuth, S. J. \& Wetton, B. T. R. 1995 Implicit explicit methods for timedependent partial-differential equations. Siam Journal on Numerical Analysis 32 (3), $797-$ 823.

Badalassi, V. E., Ceniceros, H. D. \& Banerjee, S. 2003 Computation of multiphase systems with phase field models. Journal of Computational Physics 190 (2), 371-397.

Birdi, K. S., Vu, D. T. \& Winter, A. 1989 A study of the evaporation rates of small water drops placed on a solid-surface. Journal of Physical Chemistry 93 (9), 3702-3703.

Bourgès-Monnier, C. \& Shanahan, M. E. R. 1995 Influence of evaporation on contact angle. Langmuir 11 (7), 2820-2829.

Burelbach, J. P., Bankoff, S. G. \& Davis, S. H. 1988 Nonlinear stability of evaporating condensing liquid-films. Journal of Fluid Mechanics 195, 463-494.

CAhn, J. W. 1961 On spinodal decomposition. Acta Metallurgica 9 (9), 795-801.

CAHn, J. W. \& Hilliard, J. E. 1958 Free energy of a nonuniform system .1. Interfacial free energy. Journal of Chemical Physics 28 (2), 258-267.

CAhn, J. W. \& Hilliard, J. E. 1959 Free energy of a nonuniform system .3. Nucleation in a 2-component incompressible fluid. Journal of Chemical Physics 31 (3), 688-699.

Carlson, A., Do-Quang, M. \& Amberg, G. 2009 Modeling of dynamic wetting far from equilibrium. Physics of Fluids 21 (12), 121701.

Cazabat, A. M. \& Guena, G. 2010 Evaporation of macroscopic sessile droplets. Soft Matter $6(12), 2591-2612$.

Crafton, E. F. \& Black, W. Z. 2004 Heat transfer and evaporation rates of small liquid droplets on heated horizontal surfaces. International Journal of Heat and Mass Transfer 47 (6-7), 1187-1200.

David, S., Sefiane, K. \& TAdrist, L. 2007 Experimental investigation of the effect of thermal properties of the substrate in the wetting and evaporation of sessile drops. Colloids and Surfaces a-Physicochemical and Engineering Aspects 298 (1-2), 108-114.

Deegan, R. D., Bakajin, O., Dupont, T. F., Huber, G., Nagel, S. R. \& Witten, T. A. 1997 Capillary flow as the cause of ring stains from dried liquid drops. Nature 389 (6653), 827-829. 
Deegan, R. D., Bakajin, O., Dupont, T. F., Huber, G., Nagel, S. R. \& Witten, T. A. 2000 Contact line deposits in an evaporating drop. Physical Review E 62 (1), 756-765.

Ding, H., Gilani, M. N. H. \& Spelt, P. D. M. 2010 Sliding, pinch-off and detachment of a droplet on a wall in shear flow. Journal of Fluid Mechanics 644, 217-244.

Ding, H. \& Spelt, P. D. M. $2007 a$ Inertial effects in droplet spreading: a comparison between diffuse-interface and level-set simulations. Journal of Fluid Mechanics 576, 287-296.

Ding, H. \& Spelt, P. D. M. $2007 b$ Wetting condition in diffuse interface simulations of contact line motion. Physical Review E $\mathbf{7 5}$ (4), 046708.

Ding, H. \& Spelt, P. D. M. 2008 Onset of motion of a three-dimensional droplet on a wall in shear flow at moderate Reynolds numbers. Journal of Fluid Mechanics 599, 341-362.

Ding, H., Spelt, P. D. M. \& Shu, C. 2007 Diffuse interface model for incompressible two-phase flows with large density ratios. Journal of Computational Physics 226 (2), 2078-2095.

Dunn, G. J., Wilson, S. K., Duffy, B. R., David, S. \& Sefiane, K. 2009 The strong influence of substrate conductivity on droplet evaporation. Journal of Fluid Mechanics 623, 329-351.

ERBIL, H. Y. 2012 Evaporation of pure liquid sessile and spherical suspended drops: A review. Advances in Colloid and Interface Science 170 (1-2), 67-86.

Erbil, H. Y., McHale, G. \& Newton, M. I. 2002 Drop evaporation on solid surfaces: Constant contact angle mode. Langmuir 18 (7), 2636-2641.

FANG, G. \& WARD, C. 1999 Temperature measured close to the interface of an evaporating liquid. Physical Review E 59 (1), 417-428.

Gatapova, E. Y., Semenova, A. A., Zaitsev, D. V. \& Kabov, O. A. 2014 Evaporation of a sessile water drop on a heated surface with controlled wettability. Colloids and Surfaces a-Physicochemical and Engineering Aspects 441, 776-785.

Girard, F. \& ANTONI, M. 2008 Influence of substrate heating on the evaporation dynamics of pinned water droplets. Langmuir 24 (20), 11342-11345.

Girard, F., Antoni, M., Faure, S. \& Steinchen, A. 2006 Evaporation and Marangoni driven convection in small heated water droplets. Langmuir 22 (26), 11085-11091.

Girard, F., Antoni, M., Faure, S. \& Steinchen, A. 2008 a Influence of heating temperature and relative humidity in the evaporation of pinned droplets. Colloids and Surfaces a-Physicochemical and Engineering Aspects 323 (1-3), 36-49.

Girard, F., Antoni, M. \& Sefiane, K. $2008 b$ On the effect of Marangoni flow on evaporation rates of heated water drops. Langmuir 24 (17), 9207-9210.

Hu, H. \& Larson, R. G. 2002 Evaporation of a sessile droplet on a substrate. Journal of Physical Chemistry B 106 (6), 1334-1344.

Hu, H. \& Larson, R. G. 2005 a Analysis of the effects of Marangoni stresses on the microflow in an evaporating sessile droplet. Langmuir 21 (9), 3972-3980.

Hu, H. \& LARSOn, R. G. $2005 b$ Analysis of the microfluid flow in an evaporating sessile droplet. Langmuir 21 (9), 3963-3971.

Hu, H. \& Larson, R. G. 2006 Marangoni effect reverses coffee-ring depositions. Journal of Physical Chemistry B 110 (14), 7090-7094.

JACQMin, D. 1999 Calculation of two-phase Navier-Stokes flows using phase-field modeling. Journal of Computational Physics 155 (1), 96-127.

JACQMin, D. 2000 Contact-line dynamics of a diffuse fluid interface. Journal of Fluid Mechanics 402, $57-88$.

Jansen, H. P., Zandvliet, H. J. W. \& KooiJ, E. S. 2014 Evaporation of elongated droplets on chemically stripe-patterned surfaces. International Journal of Heat and Mass Transfer (In press) .

Karapetsas, G., Matar, O. K., Valluri, P. \& Sefiane, K. 2012 Convective rolls and hydrothermal waves in evaporating sessile drops. Langmuir 28 (31), 11433-11439.

Kim, T. H., Kommer, E., Dessiatoun, S. \& Kim, J. 2012 Measurement of two-phase flow and heat transfer parameters using infrared thermometry. International Journal of Multiphase Flow 40, 56-67.

Liu, X. D., Osher, S. \& Chan, T. 1994 Weighted Essentially Nonoscillatory Schemes. Journal of Computational Physics 115 (1), 200-212.

Moffat, J. R., Sefiane, K. \& Shanahan, M. E. R. 2009 Effect of TiO2 nanoparticles on 
contact line stick-slip behavior of volatile drops. Journal of Physical Chemistry B 113 (26), 8860-8866.

Mollaret, R., Sefiane, K., Christy, J. R. E. \& Veyret, D. 2004 Experimental and numerical investigation of the evaporation into air of a drop on a heated surface. Chemical Engineering Research \& Design 82 (A4), 471-480.

Murisic, N \& Kondic, L 2011 On evaporation of sessile drops with moving contact lines. Journal of Fluid Mechanics 679, 219-246.

Nguyen, T. A. H. \& Nguyen, A. V. 2012 Increased evaporation kinetics of sessile droplets by using nanoparticles. Langmuir 28 (49), 16725-16728.

Nguyen, T. A. H., Nguyen, A. V., Hampton, M. A., Xu, Z. P., Huang, L. B. \& Rudolph, V. 2012 Theoretical and experimental analysis of droplet evaporation on solid surfaces. Chemical Engineering Science 69 (1), 522-529.

Orejon, D., Sefiane, K. \& Shanahan, M. E. R. 2011 Stick-slip of evaporating droplets: Substrate hydrophobicity and nanoparticle concentration. Langmuir 27 (21), 12834-12843.

Picknett, R. G. \& Bexon, R. 1977 Evaporation of sessile or pendant drops in still air. Journal of Colloid and Interface Science 61 (2), 336-350.

Poulard, C., Benichou, O. \& Cazabat, A. M. 2003 Freely receding evaporating droplets. Langmuir 19 (21), 8828-8834.

Qian, T. Z., WAng, X. P. \& Sheng, P. 2006 A variational approach to moving contact line hydrodynamics. Journal of Fluid Mechanics 564, 333-360.

Ristenpart, W. D., Kim, P. G., Domingues, C., Wan, J. \& Stone, H. A. 2007 Influence of substrate conductivity on circulation reversal in evaporating drops. Physical Review Letters 99 (23), 234502.

Rowan, S. M., Newton, M. I. \& Mchale, G. 1995 Evaporation of microdroplets and the wetting of solid-surfaces. Journal of Physical Chemistry 99 (35), 13268-13271.

Ruiz, O. E. \& Black, W. Z. 2002 Evaporation of water droplets placed on a heated horizontal surface. Journal of Heat Transfer-Transactions of the Asme 124 (5), 854-863.

Sáenz, P. J., Valluri, P., Sefiane, K., Karapetsas, G. \& Matar, O. K. 2014 On phase change in Marangoni-driven flows and its effects on the hydrothermal-wave instabilities. Physics of Fluids $\mathbf{2 6}$ (2), 024114.

Sefiane, K. \& Bennacer, R. 2011 An expression for droplet evaporation incorporating thermal effects. Journal of Fluid Mechanics 667, 260-271.

Sefiane, K., Moffat, J. R., Matar, O. K. \& Craster, R. V. 2008 Self-excited hydrothermal waves in evaporating sessile drops. Applied Physics Letters 93 (7), 074103.

Sefiane, K. \& TAdrist, L. 2006 Experimental investigation of the de-pinning phenomenon on rough surfaces of volatile drops. International Communications in Heat and Mass Transfer 33 (4), 482-490.

Sefiane, K., Wilson, S. K., David, S., Dunn, G. J. \& Duffy, B. R. 2009 On the effect of the atmosphere on the evaporation of sessile droplets of water. Physics of Fluids 21 (6), 062101-1 - 062101-9.

SePpeCher, P. 1996 Moving contact lines in the Cahn-Hilliard theory. International Journal of Engineering Science 34 (9), 977-992.

Shanahan, M. E. R. 1995 Simple theory of stick-slip wetting hysteresis. Langmuir 11 (3), 1041-1043.

Shanahan, M. E. R., Sefiane, K. \& Moffat, J. R. 2011 Dependence of volatile droplet lifetime on the hydrophobicity of the substrate. Langmuir 27 (8), 4572-4577.

Sobac, B. \& Brutin, D. 2012 Thermal effects of the substrate on water droplet evaporation. Physical Review E 86 (2).

Stauber, J. M., Wilson, S. K., Duffy, B. R. \& Sefiane, K. 2014 On the lifetimes of evaporating droplets. Journal of Fluid Mechanics 744, R2.

Sui, Y., Ding, H. \& Spelt, P. D. M. 2014 Numerical simulations of flows with moving contact lines. Annual Review of Fluid Mechanics 46 (1), 97-119.

Valluri, P., Naraigh, L. O., Ding, H. \& Spelt, P. D. M. 2010 Linear and nonlinear spatiotemporal instability in laminar two-layer flows. Journal of Fluid Mechanics 656, 458-480.

VAN DER WAALS, J. D. 1979 The thermodynamic theory of capillarity under the hypothesis of a continuous variation of density. Journal of Statistical Physics 20 (2), 197-200. 
WARD, C. \& FAnG, G. 1999 Expression for predicting liquid evaporation flux: Statistical rate theory approach. Physical Review E 59 (1), 429-440.

Xu, X. F. \& LuO, J. B. 2007 Marangoni flow in an evaporating water droplet. Applied Physics Letters 91 (12), 124102.

YAng, K., Hong, F. \& Cheng, P. 2014 A fully coupled numerical simulation of sessile droplet evaporation using Arbitrary LagrangianEulerian formulation. International Journal of Heat and Mass Transfer 70, 409-420.

Yue, P. T., Zhou, C. F. \& Feng, J. J. 2010 Sharp-interface limit of the Cahn-Hilliard model for moving contact lines. Journal of Fluid Mechanics 645, 279-294. 